Sensorl ess par anet er est i mat $i$ on of el ect romagnet i c transducer consi deri ng eddy currents

\begin{tabular}{|l|l|}
\hline 著者 & $\begin{array}{l}\text { I kegane Tor u, Takagi Kent ar o, I noue Tsuyoshi , } \\
\text { Ji kuya I chi ro }\end{array}$ \\
\hline $\begin{array}{l}\text { j our nal or } \\
\text { publ i cat i on ti tl e }\end{array}$ & Mechat r oni cs \\
\hline vol une & 45 \\
\hline page range & $130-142$ \\
\hline year & 2017- 08-01 \\
\hline URL & ht t p: //hdl . handl e. net /2297/48433 \\
\hline
\end{tabular}




\title{
Sensorless Parameter Estimation of Electromagnetic Transducer Considering Eddy Currents
}

\author{
Toru Ikegame $^{\mathrm{a}, *}$, Kentaro Takagi ${ }^{\mathrm{a}}$, Tsuyoshi Inoue ${ }^{\mathrm{a}}$, Ichiro Jikuya ${ }^{\mathrm{b}}$ \\ ${ }^{a}$ Department of Mechanical Science and Engineering, Nagoya University, Furo-cho, \\ Chikusa-ku, Nagoya, 464-8603 Japan. \\ ${ }^{b}$ Faculty of Electrical and Computer Engineering, Kanazawa University, Kakuma-machi, \\ Kanazawa, 920-1192, Japan
}

\begin{abstract}
This paper presents a method of estimating the parameters of an electromagnetic transducer without sensors. The proposed method utilizes the measured admittance of the electromagnetic transducer, and therefore position, velocity, and/or acceleration sensors are not necessary in this framework. Novel impedance models are proposed based on the basic physical principles of electromagnetics; in particular, the effect of eddy currents has been included in these proposed models. The validity of the proposed estimation method and models was experimentally demonstrated by comparing the parameter estimation and vibration control capabilities of the proposed models with three conventional models.
\end{abstract}

Keywords: Electromagnetic transducer, Parameter estimation, Modeling, Eddy currents, Shunt damping

\section{Introduction}

An electromagnetic transducer can act as an actuator by utilizing the Lorentz force generated from the current flowing through the magnetic field $[1,2,3]$. It can also act as a sensor by utilizing the motional electromotive force generated

\footnotetext{
${ }^{*}$ Corresponding author

Email addresses: t_ikegame@nuem.nagoya-u.ac.jp (Toru Ikegame), takagi@nuem.nagoya-u.ac.jp (Kentaro Takagi), inoue@nuem.nagoya-u.ac.jp (Tsuyoshi Inoue), jikuya@se.kanazawa-u.ac.jp (Ichiro Jikuya)

Preprint submitted to Mechatronics 
from the motion of a coil in the magnetic field [4]. Application examples of the electromagnetic transducer include but are not limited to position control $[5,6]$, sound amplification using loudspeakers [7, 8, 9, 10, 11], vibration isolation [12], valve actuation [13], load support magnetic bearings [14], magnetic levitation [15], vehicle suspension [16, 17, 18], and energy harvesting [19, 20, 21].

Electromagnetic shunt damping is an interesting technique that makes use of actuation and sensing capabilities simultaneously [22, 23, 24, 25, 26, 27, 28, 29, $30,31,32,33,34,35,36,37]$. This technique uses a shunted circuit connected across the terminals of an electromagnetic transducer for vibration control and does not use position, velocity, and/or acceleration sensors. In previous studies $[22,23]$, the resonant shunt circuit is designed to be analogous to a dynamic absorber, which is effective in providing nominal performance but is fragile when system parameters such as the natural frequency vary. Hence, precise modeling and accurate parameter estimation are crucial for the design of the shunt circuit.

In previous works, the electrical system in the electromagnetic transducer has been simply modeled by a series connection of the impedance in the coil, the internal resistance, and the motional electromotive force in the coil. The impedance in the coil is typically modeled by the self-inductance $[15,16,20,21$, $22,23,24,25,26,27,28,29,30,31,32,33,34,35,36,37]$. Among the previously published studies, a few consider eddy currents to model the impedance either 25 by adding a shunt resistance in parallel to the self-inductance $[8,9]$ or by the distributed model presented in $[10,11]$. However, these impedance models do not fully represent the measurement data, as will be shown in this paper, and practical applications continue to require more accurate impedance models, such as that presented here.

30

This paper presents a method for the sensorless parameter estimation of an electromagnetic transducer; in this method, the parameters of the mechanical, electromechanical coupling, and electrical system models are simultaneously estimated. This framework is consistent with that of electromagnetic shunt damping. The terminal voltage and current of the electromagnetic transducer are measured; specifically, the admittance is measured using an LCR meter 
or an impedance analyzer, but position, velocity, and/or acceleration sensors are not used. The parameter estimation is formulated by the weighted nonlinear least-squares method; in addition, the selection of the initial estimates for the numerical optimization is discussed in detail. The preliminary idea of this 40 paper has been presented in [38, 39], in which the method for the sensorless parameter estimation of an electromagnetic transducer is introduced only for the self-inductance to construct the impedance model. Inspired by the previous works $[8,9,10,11,40]$, the present paper introduces novel impedance models that consider eddy currents. The basic physical principles of electromagnetics, such as Ampere's circuital law, Gauss's law for magnetism, Faraday's law of induction, and Ohm's law are applied to model the impedance, and then, the partial differential equation with variable coefficients is obtained. It is difficult to convert this equation to a transfer function, and therefore, two types of physical simplifications are considered. One impedance model is derived by using spatially uniform parameters and spatially distributed eddy currents. The other impedance model is derived by using spatially nonuniform parameters and spatially lumped eddy currents. The effectiveness of the proposed parameter estimation method and the validity of the proposed models are demonstrated by performing experiments related to both parameter estimation and shunted vibration control, comparing the results obtained by the proposed method with those obtained by three conventional models, as described below.

The remainder of this paper is organized as follows. We start by presenting an overview of the conventional modeling techniques of an electromagnetic transducer for shunted vibration control and then propose novel impedance models that consider eddy currents in Section 2. We then proceed to describe the development of the parameter estimation method in Section 3. We report the demonstration of the proposed parameter estimation method through experiments in comparison with those of the conventional models in Section 4. In Section 5, we present the application of the parameter estimation results to shunted vibration control experiments to evaluate the proposed models and the accuracy of parameter estimation. Finally, we make some concluding remarks 
in Section 6.

\section{Modeling}

\subsection{Conventional modeling technique for a composite electromechanical system}

This subsection introduces a conventional modeling technique for the design and analysis of a composite electromechanical system. The composite system consists of three subsystems: a mechanical system, an electromechanical coupling system, and an electrical system.

In many vibration control systems, a mechanical system is modeled by a simple mass-spring-damper system coupled to an electromagnetic transducer $[22,31,38,39]$, as shown in Fig. 1 . The equation of motion is given by

$$
m \frac{d^{2} x}{d t^{2}}+c \frac{d x}{d t}+k x(t)=f_{d}(t)+f_{l}(t)
$$

where $m[\mathrm{~kg}]$ is the mass, $c[\mathrm{Ns} / \mathrm{m}]$ is the damping coefficient, $k[\mathrm{~N} / \mathrm{m}]$ is the spring constant, $x(t)[\mathrm{m}]$ is the displacement of the mass-spring-damper system, $f_{l}(t)[\mathrm{N}]$ is the Lorentz force generated from the electromagnetic transducer, and $f_{d}(t)[\mathrm{N}]$ is the disturbance force.

An electromechanical coupling system is given by the following pair of equations under suitable assumptions $[22,23,31,36,38,39]$ :

$$
\begin{aligned}
f_{l}(t) & =\phi i_{e}(t), \\
v_{\mathrm{emf}}(t) & =\phi \dot{x}(t),
\end{aligned}
$$

where $\phi[\mathrm{N} / \mathrm{A}$ or $\mathrm{Vs} / \mathrm{m}]$ is the electromechanical coupling coefficient, $i_{e}(t)[\mathrm{A}]$ is the current flowing through the electromagnetic transducer, and $v_{\mathrm{emf}}(t)[\mathrm{V}]$ is the motional electromotive force.

An electrical system is modeled by the series connection of the impedance of the coil, $Z_{c}(s)[\Omega]$, internal resistance in the coil, $R_{0}[\Omega]$, and motional electromotive force, $v_{\mathrm{emf}}(t)$, as shown in Fig. 2. The circuit equation is given by

$$
v_{e}(t)-v_{\text {emf }}(t)=R_{0} i_{e}(t)+v_{c}(t),
$$




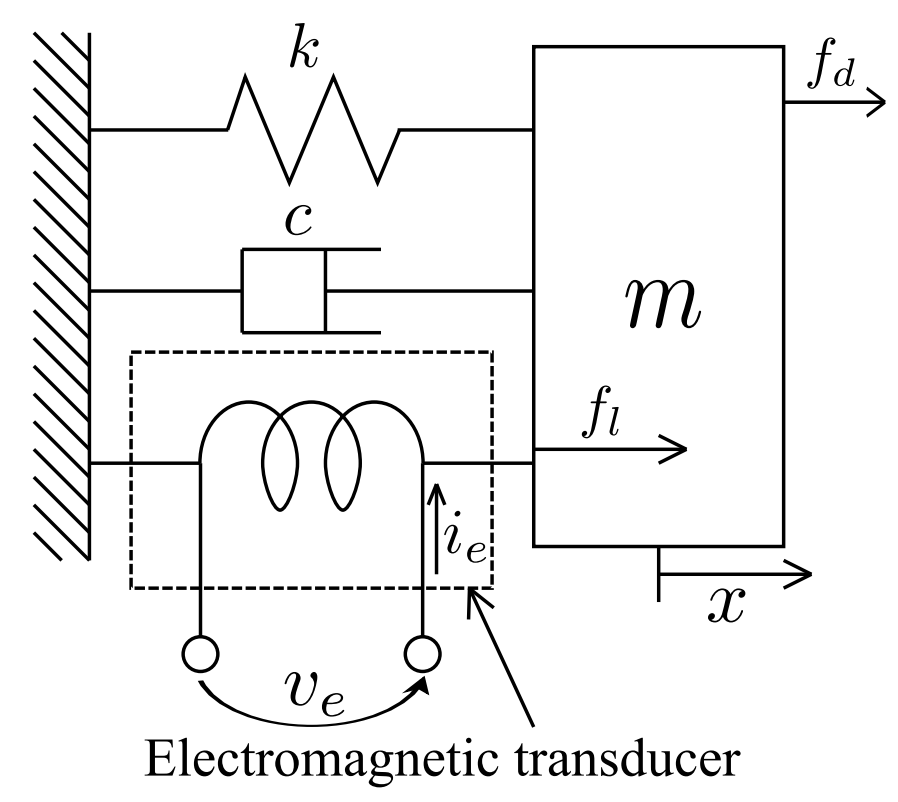

Figure 1: Simple mass-spring-damper system coupled to an electromagnetic transducer [38].

where $v_{e}(t)[\mathrm{V}]$ is the voltage across the electromagnetic transducer, and $v_{c}(t)$ $[\mathrm{V}]$ is the voltage across $Z_{c}(s)$. By taking the Laplace transformation, the impedance of the coil, $Z_{c}(s)[\Omega]$, is defined by

$$
Z_{c}(s):=\frac{\tilde{v}_{c}(s)}{\tilde{i}_{e}(s)}
$$

where the tilde represents a signal in the Laplace domain.

The impedance of the coil, $Z_{c}(s)[\Omega]$, has been typically modeled by the selfinductance, $L_{0}[\mathrm{H}]$, in $[15,16,20,21,22,23,24,25,26,27,28,29,30,31,32$, $33,34,35,36,37]$. In order to distinguish from the other impedance models, the impedance model

$$
Z_{c}^{\mathrm{I}}(s)=L_{0} s
$$

is hereafter called the conventional model $I$. The superscript in $Z_{c}(s)$ indicates the name of the models such as the conventional models or proposed models, as will be shown later.

In addition, the impedance in the coil has also been modeled by adding a shunt resistance, $R_{\mu}$, in parallel to the self-inductance, $L_{0}$, in $[8,9]$. This 


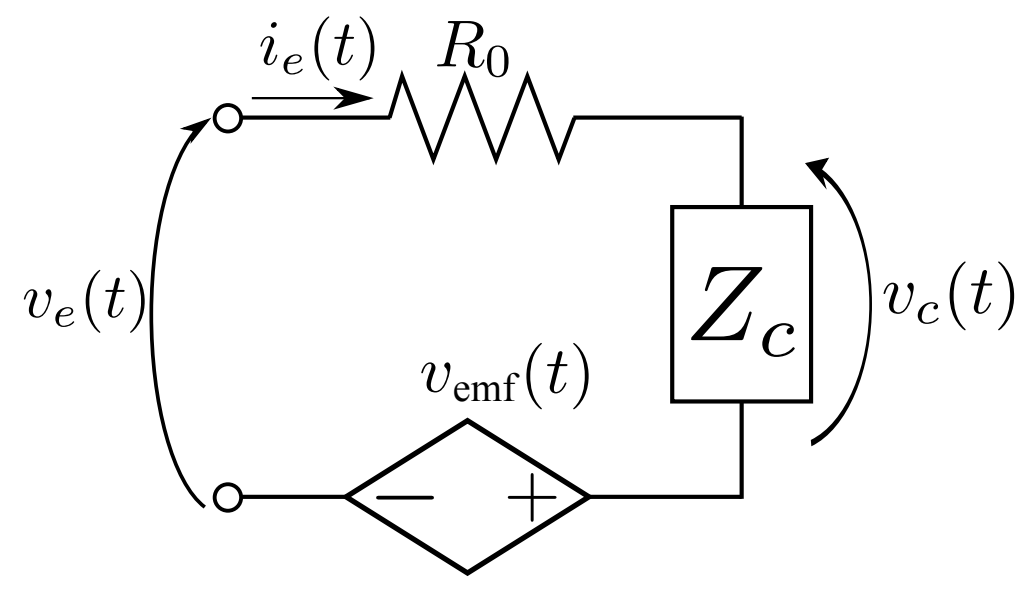

Figure 2: Electrical system model of the series connection of the impedance of the coil, $Z_{c}(s)$, the internal resistance in the coil, $R_{0}$, and the motional electromotive force, $v_{\mathrm{emf}}(t)$.

shunt resistance was considered to be largely due to eddy current loss [8]. This impedance model, which is hereinafter called the conventional model II, is then given by

$$
Z_{c}^{\mathrm{II}}(s)=\frac{R_{\mu} L_{0} s}{L_{0} s+R_{\mu}}
$$

\subsection{Impedance modeling considering eddy currents}

\subsubsection{Spatially nonuniform parameters and spatially distributed eddy currents}

Although the conventional model I is widely recognized in previous works, this model neglects the electrical conductivity of the iron core. This approximation may be valid in a low-frequency range, but is not valid in a high-frequency range because of the eddy currents induced within the iron core, which are generated by the time-varying magnetic flux in the iron core [41]. In the conventional model II, the eddy current is lumped, whereas the eddy currents are typically recognized as spatially distributed $[10,41]$. In fact, the experimental results that will be presented in Section 4 indicate that the conventional models I and II are not consistent with the actual frequency responses in mid- and high-frequency ranges. 


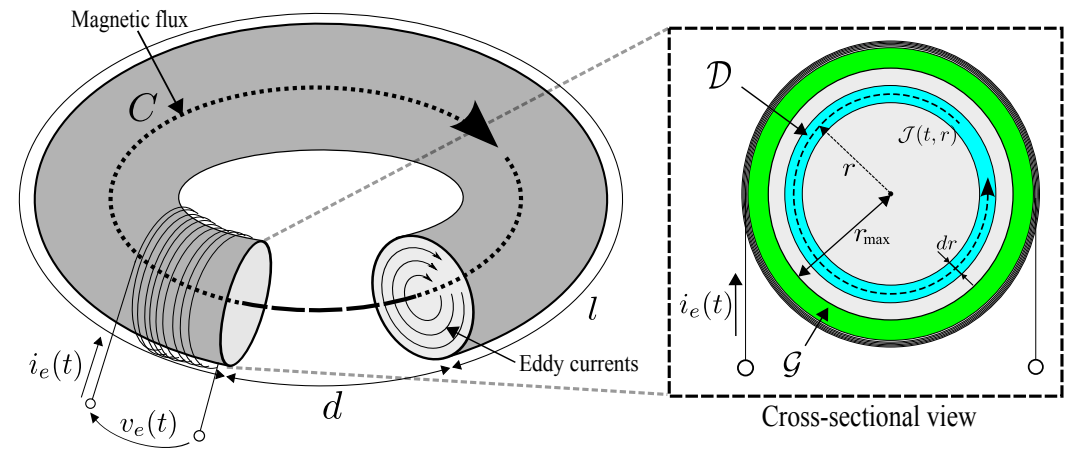

Figure 3: Schematic of a simple electromagnetic transducer modeled by pairing a toroidal iron core and a coil. The dashed box shows the schematic of the cross section of the coil and iron core with the infinitesimal cylindrical elements, $\mathcal{D}$, and with the circumferential gap, $\mathcal{G}$, between the coil and iron core. The circumferential gap is critical to the model because the coil moves relative to the iron core.

This subsection presents a novel approach for modeling $Z_{c}(s)$ by considering the spatially nonuniform parameters and spatially distributed eddy currents within the iron core. For simplicity of analysis, the electromagnetic transducer is modeled by a pair of a toroidal iron core and a coil, as shown in Fig. 3. $N_{c}$ is the number of turns of the coil around the toroidal iron core. The effect of the permanent magnet in the electromagnetic transducer is negligible in the following modeling procedure because $v_{c}(t)$ is not affected by the direct-current component of the magnetic flux according to Faraday's law of induction. Then, the eddy currents flow circularly within the iron core, in planes perpendicular to the magnetic flux. For further simplicity of analysis, the iron core is divided into infinitesimal cylindrical elements in the region along the magnetic flux, and an infinitesimal cylindrical element, $\mathcal{D}$, with the radius of $r$ is selected, as shown in Fig. 3.

Next, $Z_{c}(s)$ is derived based on the basic physical principles of electromagnetics $[42,43]$. According to Gauss's law for magnetism, the magnetic flux in the iron core and that in the gap are continuously connected. According to Ampere's circuital law, a line integral of the magnetic field passing though $\mathcal{D}$ around a closed curve, $C$, is proportional to the total current passing through 
a surface enclosed by the closed curve:

$$
\sigma(r) B(t, r)=N_{c} i_{e}(t)+\int_{r}^{r_{\max }} \mathcal{J}(t, \xi) l(\xi) d \xi, \sigma(r):=\frac{l(r)}{\mu_{0} \mu_{s}(r)}+\frac{d(r)}{\mu_{0}},
$$

where $B(t, r)[\mathrm{T}]$ is the magnetic flux density, $\mathcal{J}(t, r)\left[\mathrm{A} / \mathrm{m}^{2}\right]$ is the eddy current density flowing in $\mathcal{D}$ along the circumferential direction, $\mu_{0}[\mathrm{H} / \mathrm{m}]$ is the magnetic permeability of vacuum, $\mu_{s}(r)[-]$ is the relative magnetic permeability, $l(r)$ $[\mathrm{m}]$ and $d(r)[\mathrm{m}]$ are the magnetic path lengths of the iron core and the air gap in the axial direction respectively, and $r_{\max }[\mathrm{m}]$ is the maximum radius of the iron core. According to Faraday's law of induction, the induced electromotive force, $\mathcal{E}(t, r)[\mathrm{V}]$, in $\mathcal{D}$ along the circumferential direction is determined from the time derivative of the total magnetic flux inside $\mathcal{D}$ and is therefore given by

$$
\mathcal{E}(t, r)=-\frac{\partial}{\partial t} \int_{0}^{r} 2 \pi \xi B(t, \xi) d \xi
$$

According to Ohm's law, $\mathcal{J}(t, r)$ is also given by

$$
\mathcal{J}(t, r)=\frac{\mathcal{E}(t, r)}{2 \pi \rho(r) r}
$$

where $\rho(r)[\Omega \mathrm{m}]$ is the electrical resistivity of the iron core. It follows from (8), (9), and (10) that $B(t, r)$ satisfies the following partial differential equation:

$$
\frac{\partial B(t, r)}{\partial t}=\frac{1}{r} \frac{\partial}{\partial r}\left(\frac{\rho(r) r}{l(r)} \frac{\partial \sigma(r) B(t, r)}{\partial r}\right)
$$

with the boundary conditions:

$$
\begin{array}{r}
\sigma\left(r_{\max }\right) B\left(t, r_{\max }\right)=N_{c} i_{e}(t), \\
\lim _{r \rightarrow 0+} r \frac{\partial \sigma(r) B(t, r)}{\partial r}=0 .
\end{array}
$$

Note that a circumferential gap, $\mathcal{G}$, exists between the coil and iron core because the coil moves relative to the iron core, as shown in Fig. 3; in addition, the coil is wound around a plastic cylinder in the following experimental setup shown in Fig. 8. Eddy currents are not generated in $\mathcal{G}$, and therefore, the magnetic flux density $B_{g}(t)=N_{c} i_{e}(t) / \sigma_{g}, \sigma_{g}:=\left(l_{g}+d_{g}\right) / \mu_{0}$ is generated in $\mathcal{G}$, where the subscript $g$ in the variable or constant indicates that the symbol is related to the 
circumferential gap. According to Faraday's law of induction, the electromotive force, $v_{c}(t)$, is given by

$$
v_{c}(t)=N_{c} \frac{d}{d t}\left(\frac{N_{c} S_{g}}{\sigma_{g}} i_{e}(t)+\int_{0}^{r_{\max }} 2 \pi \xi B(t, \xi) d \xi\right),
$$

where $S_{g}\left[\mathrm{~m}^{2}\right]$ is the cross-sectional area of $\mathcal{G}$. In summary, the governing equation is the partial differential equation in (11), the boundary conditions are given by (12) and (13), and the output equation is given by (14).

\subsubsection{Spatially uniform parameters and spatially distributed eddy currents}

Because it is difficult to convert (11) to a transfer function, we assume that the physical parameters $\sigma(r), \rho(r)$, and $l(r)$ are the positive constants $\sigma, \rho$, and $l$, respectively, in this subsection. This assumption indicates that the material of the iron core is uniform and the magnetic flux leakage is negligible. Based on this assumption, (11), (12), and (13) are rewritten as follows:

$$
\begin{aligned}
& \frac{\partial B(t, r)}{\partial t}=\frac{\sigma \rho}{l} \frac{1}{r} \frac{\partial}{\partial r}\left(r \frac{\partial B(t, r)}{\partial r}\right) . \\
& \sigma B\left(t, r_{\max }\right)=N_{c} i_{e}(t), \\
& \lim _{r \rightarrow 0+} r \frac{\partial B(t, r)}{\partial r}=0,
\end{aligned}
$$

By taking the Laplace transformation of (15) with respect to $t$, this equation is reduced to an ordinary differential equation with respect to $r$.

$$
s \tilde{B}(s, r)=\frac{\sigma \rho}{l} \frac{1}{r} \frac{\partial}{\partial r}\left(r \frac{\partial \tilde{B}(s, r)}{\partial r}\right),
$$

where $\tilde{B}(s, r)$ is the Laplace transform of $B(t, r)$. By combining the ordinary differential equation in (18) with the boundary conditions in (16) and (17), the solution of the ordinary differential equation is given by

$$
\tilde{B}(s, r)=\beta^{\prime} \frac{J_{0}\left(\sqrt{-\alpha^{\prime} r^{2} s}\right)}{J_{0}\left(\sqrt{-\alpha^{\prime} r_{\max }^{2} s}\right)} \tilde{i}_{e}(s),
$$

where $\alpha^{\prime}:=l /(\sigma \rho), \beta^{\prime}:=N_{c} / \sigma$, and $J_{\nu}(z)$ are the Bessel functions of the first kind for a real number $\nu$. By taking the Laplace transformation of (14) and 
substituting (19) into the transformed equation of (14), the electromotive force in the Laplace domain, $\tilde{v}_{c}(s)$, is given by

$$
\tilde{v}_{c}(s)=\left(L_{g} s-\beta \sqrt{-s} \frac{J_{1}(\sqrt{-\alpha s})}{J_{0}(\sqrt{-\alpha s})}\right) \tilde{i}_{e}(s),
$$

where $L_{g}:=N_{c}^{2} S_{g} / \sigma_{g}, \alpha:=l r_{\max }^{2} /(\sigma \rho)$, and $\beta:=2 \pi N_{c}^{2} r_{\max } \sqrt{\rho /(\sigma l)}$ are positive constants. Now, the model of the impedance that considers spatially Uniform parameters and spatially Distributed eddy currents, $Z_{c}^{\mathrm{UD}}(s)$, which is called the $U D$ model in short, is given by

$$
Z_{c}^{\mathrm{UD}}(s)=L_{g} s-\beta \sqrt{-s} \frac{J_{1}(\sqrt{-\alpha s})}{J_{0}(\sqrt{-\alpha s})} .
$$

In this model, $L_{g}, \alpha$, and $\beta$ are unknown parameters, which will be determined 115 by performing experiments, and the number of unknown parameters is three.

Distributed modeling has also been discussed in [10,11]. The conventional model of $Z_{c}(s)$ presented in these works, which is hereinafter called the conventional model III, is given by

$$
Z_{c}^{\mathrm{III}}(s)=-\beta \sqrt{-s} \frac{J_{1}(\sqrt{-\alpha s})}{J_{0}(\sqrt{-\alpha s})} .
$$

From (22), it can be seen that the conventional model III does not include $L_{g} s$, which represents the self-inductance component caused by the circumferential gap, $\mathcal{G}$, shown in Fig. 3. Therefore, the UD model is a natural extension of the conventional model III. Moreover, the UD model is considered to be more suitable than the conventional model III because the UD model includes the gap between the coil and iron core, which is critically important to relative motion.

\subsubsection{Spatially nonuniform parameters and spatially lumped eddy currents}

In mid- and high-frequency ranges, the UD model better matches the experimental results of frequency responses than the conventional models, as will responses in the mid-frequency range. This mismatch may be caused by the assumption made in Section 2.2.2 for the UD model. 


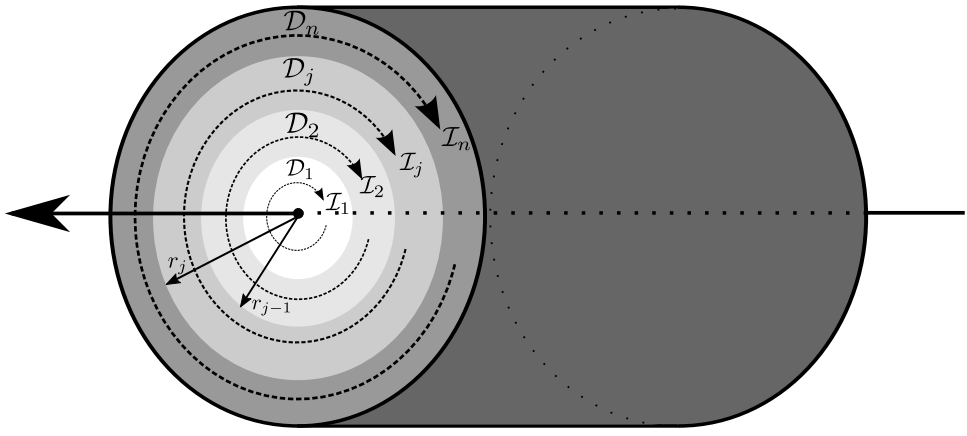

Figure 4: Schematic of the cross section of the iron core divided into $n$ rings, where $\mathcal{D}_{j}$ denotes the $j$-th ring numbered from the inside.

This subsection presents another novel approach for modeling $Z_{c}(s)$ by discretizing the model described in Section 2.2.1. The cross section of the iron core is supposed to be divided into $n$ rings, and $\mathcal{D}_{j}, j=1, \cdots, n$ denotes the $j$-th ring numbered from inside, as shown in Fig. 4. The magnetic flux in $\mathcal{D}_{j}, \Phi_{j}(t)$ $[\mathrm{Wb}]$, is defined by

$$
\Phi_{j}(t):=\int_{r_{j-1}}^{r_{j}} 2 \pi \xi B(t, \xi) d \xi
$$

By substituting (8) into (23), we have

$$
\Phi_{j}(t)=N_{c} \int_{r_{j-1}}^{r_{j}} \frac{2 \pi \xi}{\sigma(\xi)} d \xi i_{e}(t)+\int_{r_{j-1}}^{r_{j}} \frac{2 \pi \xi}{\sigma(\xi)} \int_{\xi}^{r_{\max }} \mathcal{J}\left(t, \xi^{\prime}\right) l\left(\xi^{\prime}\right) d \xi^{\prime} d \xi
$$

By approximating the interval of integration $\left[\xi, r_{\max }\right]$ as $\left[r_{j-1}, r_{\max }\right]$, we have

$$
\Phi_{j}(t)=N_{c} \mathcal{P}_{j} i_{e}(t)+\mathcal{P}_{j} \sum_{k=j}^{n} \mathcal{I}_{k}(t)
$$

where the magnetic permeance, $\mathcal{P}_{j}[\mathrm{H}]$, and the lumped eddy current, $\mathcal{I}_{j}(t)[\mathrm{A}]$, are defined by

$$
\begin{aligned}
\mathcal{P}_{j} & :=\int_{r_{j-1}}^{r_{j}} \frac{2 \pi \xi}{\sigma(\xi)} d \xi \\
\mathcal{I}_{j}(t) & :=\int_{r_{j-1}}^{r_{j}} \mathcal{J}(t, \xi) l(\xi) d \xi
\end{aligned}
$$


By substituting (23) into (9), we have

$$
\mathcal{E}\left(t, r_{j}\right)=-\frac{d}{d t} \sum_{k=1}^{j} \Phi_{k}(t) .
$$

By substituting (10) into (27), we have

$$
\mathcal{I}_{j}(t)=\int_{r_{j-1}}^{r_{j}} \frac{l(\xi) \mathcal{E}(t, \xi)}{2 \pi \rho(\xi) \xi} d \xi .
$$

By approximating $\mathcal{E}(t, \xi)$ in $(29)$ as a piecewise constant function, we have

$$
R_{j} \mathcal{I}_{j}(t)=\mathcal{E}\left(t, r_{j}\right)
$$

where the electric resistance, $R_{j}[\Omega]$, is defined by

$$
R_{j}:=\left(\int_{r_{j-1}}^{r_{j}} \frac{l(\xi)}{2 \pi \rho(\xi) \xi} d \xi\right)^{-1}
$$

By substituting (23) into (14), we have

$$
v_{c}(t)=N_{c} \frac{d}{d t}\left(\frac{N_{c} S_{g}}{\sigma_{g}} i_{e}(t)+\sum_{j=1}^{n} \Phi_{j}(t)\right) .
$$

From (25), (28), (30) and (32), the circuit equation is given in the matrix form as follows:

$$
\left[\begin{array}{c}
v_{c}(t) \\
\mathbf{0}
\end{array}\right]=\boldsymbol{L} \frac{d}{d t}\left[\begin{array}{c}
i_{e}(t) \\
\mathcal{I}(t)
\end{array}\right]+\left[\begin{array}{ll}
0 & \mathbf{0} \\
\mathbf{0} & \boldsymbol{R}
\end{array}\right]\left[\begin{array}{c}
i_{e}(t) \\
\mathcal{I}(t)
\end{array}\right]
$$


where the components of vectors and matrices are given by

$$
\begin{aligned}
& \boldsymbol{L}=\left[\begin{array}{cccc}
L_{0} & M_{01} & \cdots & M_{0 n} \\
M_{10} & L_{1} & \cdots & M_{1 n} \\
\vdots & \vdots & \ddots & \vdots \\
M_{n 0} & M_{n 1} & \cdots & L_{n}
\end{array}\right] \\
& :=\left[\begin{array}{cc}
N_{c}^{2}\left(\mathcal{P}_{g}+\boldsymbol{U}^{\mathrm{T}} \mathcal{P} \boldsymbol{U}\right) & N_{c} \boldsymbol{U}^{\mathrm{T}} \mathcal{P} \boldsymbol{D}^{\mathrm{T}} \\
N_{c} \boldsymbol{D} \mathcal{P} \boldsymbol{U} & \boldsymbol{D} \mathcal{P} \boldsymbol{D}^{\mathrm{T}}
\end{array}\right], \\
& \mathcal{P}_{g}:=\frac{S_{g}}{\sigma_{g}}=\frac{L_{g}}{N_{c}^{2}}, \\
& \mathcal{P}:=\operatorname{diag}\left[\begin{array}{lll}
\mathcal{P}_{1} & \cdots & \mathcal{P}_{n}
\end{array}\right], \\
& \boldsymbol{R}:=\operatorname{diag}\left[\begin{array}{lll}
R_{1} & \cdots & R_{n}
\end{array}\right], \\
& \mathcal{I}(t):=\left[\begin{array}{lll}
\mathcal{I}_{1}(t) & \cdots & \mathcal{I}_{n}(t)
\end{array}\right]^{\mathrm{T}}, \\
& \boldsymbol{U}:=\left[\begin{array}{lll}
1 & \cdots & 1
\end{array}\right]^{\mathrm{T}} \in \mathbb{R}^{n}, \\
& \boldsymbol{D}:=\left[\begin{array}{ccc}
1 & & \\
\vdots & \ddots & \\
1 & \cdots & 1
\end{array}\right] \in \mathbb{R}^{n \times n} .
\end{aligned}
$$

From (33), it might be possible to infer that the lumped eddy currents in (27) flow in the fictitious coils in the iron core. Figure 5 shows the equivalent circuit diagram of Fig. 4. Here, $L_{0}$ can be recognized as the self-inductance of the coil; $L_{j}, 1 \leq j \leq n$, the self-inductances of the fictitious coils in the iron core; $M_{i j}$, $0 \leq i, j \leq n$, the mutual-inductances between the coils; and $R_{j}, 1 \leq j \leq n$, the internal resistance of the fictitious coils in the iron core. Note that the number of discretization, $n$, is to be appropriately selected in modeling experiments. In addition, the magnetic energy stored in the inductor is positive and the resistivity of the iron core is positive. It follows that $\boldsymbol{L}$ is symmetric positive definite and $R_{j}$ is positive, and therefore, the circuit is passive[44]. Now, by taking the Laplace transformation of (33), the model of the impedance considering spatially Nonuniform parameters and spatially Lumped eddy currents, $Z_{c}^{\mathrm{NL}}(s)$, 


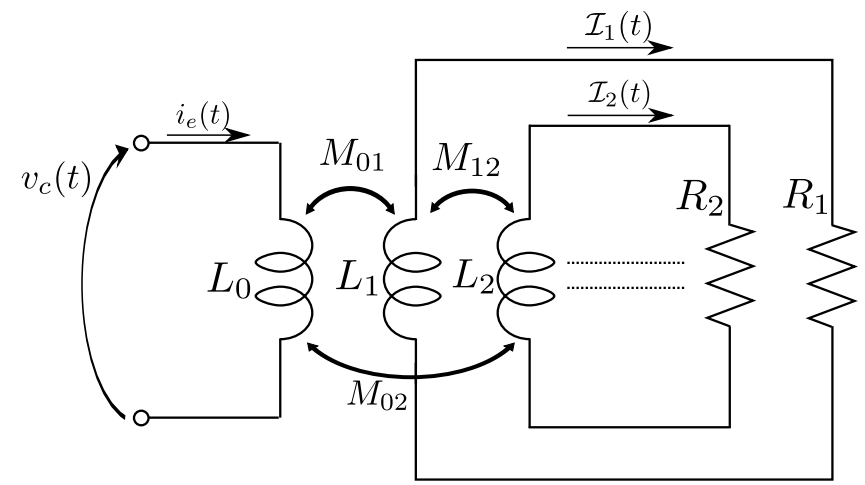

Figure 5: Equivalent circuit diagram of Fig. 4, where $L_{0}$ can be recognized as the selfinductances of the coil; $L_{j}$ are the self-inductances of the fictitious coils in the iron core; $M_{i j}$ are the mutual-inductances between the coils; and $R_{j}$ are the internal resistances of the fictitious coils in the iron core.

which is called the $N L$ model in short, is given by

$$
Z_{c}^{\mathrm{NL}}(s)=N_{c}^{2}\left(\mathcal{P}_{g}+\boldsymbol{U}^{\mathrm{T}} \mathcal{P} \boldsymbol{U}\right) s-N_{c}^{2} \boldsymbol{U}^{\mathrm{T}} \mathcal{P} \boldsymbol{D}^{\mathrm{T}}\left(\boldsymbol{D} \mathcal{P} \boldsymbol{D}^{\mathrm{T}} s+\boldsymbol{R}\right)^{-1} \boldsymbol{D} \mathcal{P} \boldsymbol{U} s^{2}
$$

In this model, $\mathcal{P}_{g}, \mathcal{P}_{j}$, and $R_{j}$ are unknown parameters, which will be determined by performing experiments, and the number of unknown parameters is $2 n+1$.

\subsubsection{Spatially uniform parameters and spatially lumped eddy currents}

Following the procedure used in Sections 2.2.1 and 2.2.2, we consider an assumption that the physical parameters $\sigma(r), \rho(r)$, and $l(r)$ in Section 2.2.3 are supposed to be positive constants $\sigma, \rho$, and $l$, respectively. From (26) and (31), we have

$$
\begin{aligned}
\mathcal{P}_{j} & =\frac{\pi}{\sigma}\left(r_{j}^{2}-r_{j-1}^{2}\right), \\
R_{j} & =\frac{2 \pi \rho r_{j}}{l\left(r_{j}-r_{j-1}\right)},
\end{aligned}
$$

where the integrand in (31) is approximated by $l / 2 \pi \rho r_{j}$. The cross-sectional areas of $\mathcal{D}_{j}$ are considered to be equal for simplicity, and then, $r_{j}$ is given by

$$
r_{j}=\sqrt{\frac{j}{n}} r_{\max } .
$$


By substituting (44) into (42) and (43), $\mathcal{P}_{j}$ and $R_{j}$ are given by

$$
\begin{aligned}
& \mathcal{P}_{j}=\frac{\pi r_{\text {max }}^{2}}{\sigma} \frac{1}{n}=\frac{\sqrt{\alpha} \beta}{2 N_{c}^{2}} \frac{1}{n}, \\
& R_{j}=\frac{2 \pi \rho}{l}(j+\sqrt{j(j-1)})=\frac{\beta}{N_{c}^{2} \sqrt{\alpha}}(j+\sqrt{j(j-1)}),
\end{aligned}
$$

where the right-hand sides of (45) and (46) are represented in terms of $\alpha$ and $\beta$. The model of the impedance considering spatially uniform parameters and spatially lumped eddy currents is obtained by substituting (35), (45), and (46) into (41). This impedance model can be recognized as the discretized form of the UD model in (21). Note that this impedance model includes three unknown parameters: $L_{g}, \alpha$, and $\beta$. The selection of the initial values for the nonlinear numerical optimization are detailed in Appendix A.

\section{Parameter estimation method}

\subsection{Appropriate range of frequency domain measurements}

This subsection discusses whether the parameters in the proposed models can be really estimated without using position, velocity, and/or acceleration sensors, i.e., by measuring $v_{e}(t)$ and $i_{e}(t)$ but without measuring $x(t)$. In the parameter estimation, it is suitable to assume that the disturbance force is zero, i.e., $f_{d}(t)=0$. By taking the Laplace transformation of (1)-(5), the admittance of the electromagnetic transducer, $Y_{e}(s)[\mathrm{S}]$, is given by

$$
Y_{e}(s):=\frac{\tilde{i}_{e}(s)}{\tilde{v}_{e}(s)}=\left(Z_{c}(s)+R_{0}+\frac{\bar{\phi}^{2} s}{s^{2}+2 \zeta \omega_{0} s+\omega_{0}^{2}}\right)^{-1},
$$

140 $c / \sqrt{4 m k}$ is the damping ratio, and $\bar{\phi}:=\phi / \sqrt{m}$ is the electromechanical coupling coefficient normalized by the mass; more specifically, $Y_{e}^{\mathrm{I}}(s), Y_{e}^{\mathrm{II}}(s), Y_{e}^{\mathrm{III}}(s)$, $Y_{e}^{\mathrm{UD}}(s)$, and $Y_{e}^{\mathrm{NL}}(s)$ are given by substituting $Z_{c}^{\mathrm{I}}(s), Z_{c}^{\mathrm{II}}(s), Z_{c}^{\mathrm{III}}(s), Z_{c}^{\mathrm{UD}}(s)$, and $Z_{c}^{\mathrm{NL}}(s)$ into $Z_{c}(s)$ in $(47)$.

145

The frequency response of the admittance, $Y_{e}(\mathrm{j} \omega)$, can be directly measured by using an LCR meter or an impedance analyzer. The problem here is to 

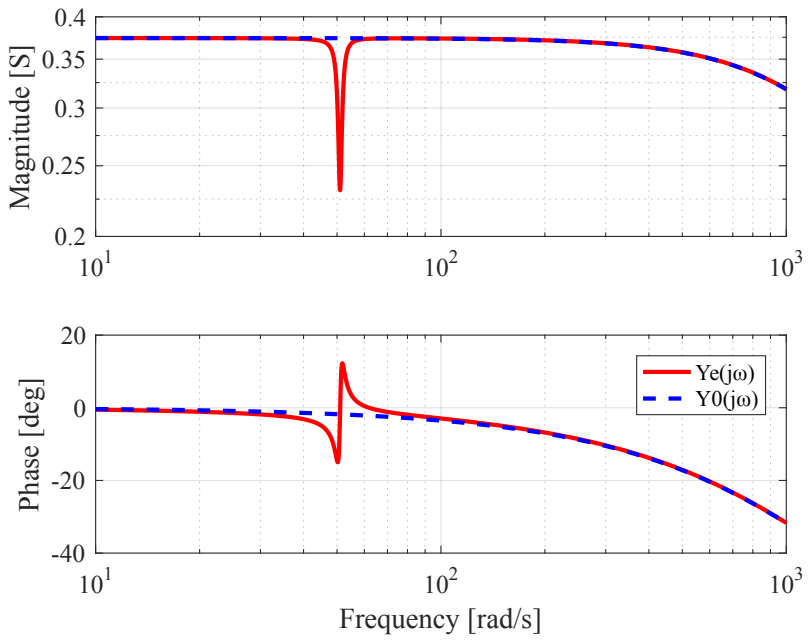

Figure 6: Bode admittance diagrams of $Y_{e}^{\mathrm{I}}(\mathrm{j} \omega)$ (red solid line) and $Y_{0}^{\mathrm{I}}(\mathrm{j} \omega)$ (blue broken line) for the parameters listed in Table 1 , where $Y_{e}^{\mathrm{I}}(\mathrm{j} \omega)$ includes the effect of the mechanical system and $Y_{0}^{\mathrm{I}}(\mathrm{j} \omega)$ does not. $Y_{e}^{\mathrm{I}}(\mathrm{j} \omega)$ and $Y_{0}^{\mathrm{I}}(\mathrm{j} \omega)$ are different in the vicinity of the natural frequency of the mechanical system, i.e., $\omega \simeq 50[\mathrm{rad} / \mathrm{s}]$, whereas they are similar outside of this region.

simultaneously estimate all the parameters of the mechanical, electromechanical coupling, and electrical systems. Let us compare $Y_{e}^{\mathrm{I}}(s)$ with $Y_{0}^{\mathrm{I}}(s):=\left(L_{0} s+\right.$ $\left.R_{0}\right)^{-1}$, which is obtained by substituting $\bar{\phi}=0$ into (47). Figure 6 illustrates the Bode diagrams of $Y_{e}^{\mathrm{I}}(\mathrm{j} \omega)$ and $Y_{0}^{\mathrm{I}}(\mathrm{j} \omega)$ for the parameters listed in Table 1 , which will be obtained later in the estimation experiments. It is interesting to observe that $Y_{e}^{\mathrm{I}}(\mathrm{j} \omega)$ and $Y_{0}^{\mathrm{I}}(\mathrm{j} \omega)$ are different in the vicinity of the natural frequency of the mechanical system, i.e., $\omega \simeq 50[\mathrm{rad} / \mathrm{s}]$, and they are similar outside, i.e., $\omega \leq 40[\mathrm{rad} / \mathrm{s}]$ or $\omega \geq 70[\mathrm{rad} / \mathrm{s}]$. Because $Y_{0}^{\mathrm{I}}(\mathrm{j} \omega)$ exhibits a low-pass characteristic, $R_{0}$ is dominant in the low-frequency range and $L_{0}$ is dominant in the high-frequency range. This suggests that the parameter estimation can be carried out from $Y_{e}(\mathrm{j} \omega)$ when $\omega$ covers a sufficiently wide range that includes the natural frequency of the mechanical system.

\subsection{Parameter estimation from frequency domain measurements}

This subsection presents a framework for estimating parameters from the frequency domain measurements of the admittance, $Y_{e}(\mathrm{j} \omega)$. The measured data are 
the complex values of the admittance denoted by $Y_{s}\left(\mathrm{j} \omega_{s}[k]\right), k=1,2, \cdots, N_{s}$, where $\omega_{s}[k]$ is the $k$-th sampled angular frequency and $N_{s}$ is the number of sampling points. As discussed in (47), we estimate the parameters in the impedance model, given by (6), (7), (22), (21), or (41), as well as $\omega_{0}, \zeta, \bar{\phi}$, and $R_{0}$. The parameters can be written in the vector form for each impedance model as follows:

$$
\boldsymbol{\theta}_{\text {nls }}:= \begin{cases}{\left[\zeta, \omega_{0}, \bar{\phi}, R_{0}, L_{0}\right]^{\mathrm{T}}} & \text { Eq. (6) } \\ {\left[\zeta, \omega_{0}, \bar{\phi}, R_{0}, L_{0}, R_{\mu}\right]^{\mathrm{T}}} & \text { Eq. (7) } \\ {\left[\zeta, \omega_{0}, \bar{\phi}, R_{0}, \alpha, \beta\right]^{\mathrm{T}}} & \text { Eq. (22) } \\ {\left[\zeta, \omega_{0}, \bar{\phi}, R_{0}, L_{g}, \alpha, \beta\right]^{\mathrm{T}}} & \text { Eq. (21) } \\ {\left[\zeta, \omega_{0}, \bar{\phi}, R_{0}, \mathcal{P}_{g}, \mathcal{P}_{j}, R_{j}\right]^{\mathrm{T}},} & \\ (j=1, \cdots, n) & \text { Eq. (41) }\end{cases}
$$

Then, $Y_{e}(s)$ is rewritten as $Y_{e}\left(s ; \boldsymbol{\theta}_{\mathrm{nls}}\right)$ to express the parameter dependence. The parameter estimation problem is now formulated by the weighted nonlinear least-squares method in the frequency domain.

$$
\min _{\boldsymbol{\theta}_{\mathrm{nls}}} \sum_{k=1}^{N_{s}}\left|\left(\frac{1}{Y_{e}\left(\mathrm{j} \omega_{s}[k] ; \boldsymbol{\theta}_{\mathrm{nls}}\right)}-\frac{1}{Y_{s}\left(\mathrm{j} \omega_{s}[k]\right)}\right) W_{\mathrm{nls}}\left(\mathrm{j} \omega_{s}[k]\right)\right|^{2},
$$

160

where $W_{\mathrm{nls}}\left(\mathrm{j} \omega_{s}[k]\right)$ is the weighting function.

Considering the sensitivity of parameters to different frequency ranges, the problem in (49) is solved in two steps. Recall that $R_{0}$ is dominant in the low-frequency range and $L_{0}$ is dominant in the high-frequency range. In the first step, all the parameters, $\boldsymbol{\theta}_{\mathrm{nls}}$, are calculated by solving (49) using the weighting function $W_{\mathrm{nls}}(s)=1 / s$, and then, $R_{0}$ and $\omega_{0}$ are determined. In the second step, the other parameters are calculated by solving (49) using the weighting function $W_{\text {nls }}(s)=1$. This two-step estimation procedure is effective in improving the convergence properties of nonlinear numerical optimization in (49). The selection of the initial values for the nonlinear numerical optimization are detailed in Appendix A. 


\section{Parameter estimation experiments}

\subsection{Experimental setup}

The experimental demonstration of the proposed estimation method is described in this section. The experimental setup is common to the one in [38] ingly, the estimated value is found to be nearly $50[\mathrm{rad} / \mathrm{s}]$.

The initial estimates are determined from the weighted linear least-squares in (A.2). The measurements of $Y_{e}(\mathrm{j} \omega)$ in the range 10-300 [rad/s], which covers the natural frequency of $50[\mathrm{rad} / \mathrm{s}]$, are tested. Note that a low-frequency range estimation. In the case of the conventional model I, the initial estimates given in (26)-(30) of [38] are applied to determine $\zeta, \omega_{0}, \bar{\phi}, R_{0}$, and $L_{0}$. In the case of the conventional model II, (A.8) is applied to determine $\zeta, \omega_{0}, \bar{\phi}, R_{0}, L_{0}$, and $R_{\mu}$. In the case of the conventional model III, (A.14) is applied to determine $\zeta$, 
Electromagnetic transducer

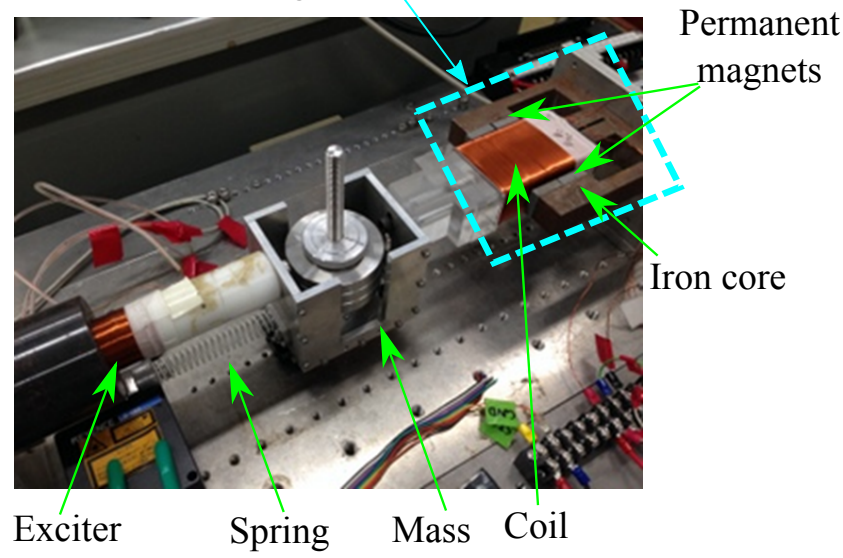

Figure 7: Photograph of the experimental setup composed an exciter, a spring, a mass, and an electromagnetic transducer comprising two permanent magnets, an iron core, and a coil [38].

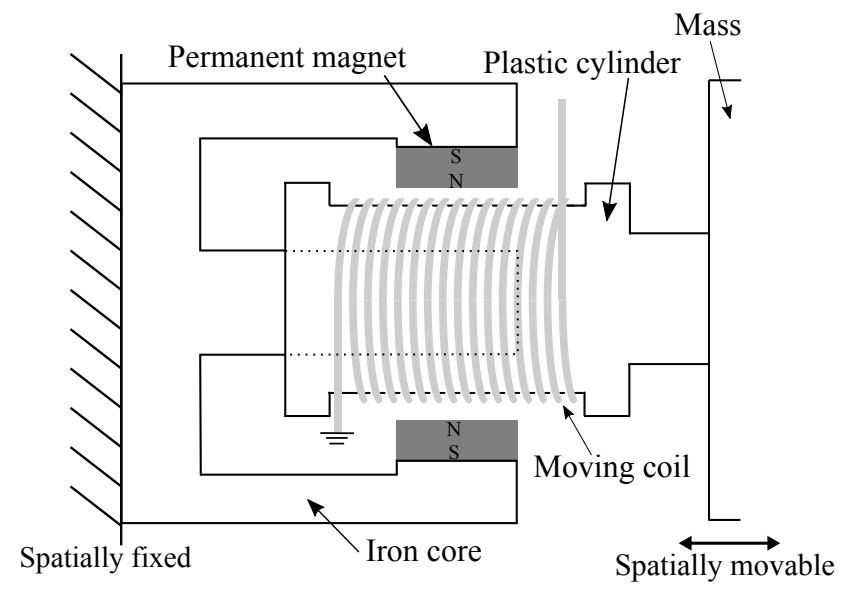

Figure 8: Schematic of an electromagnetic transducer comprising a moving coil, two permanent magnets, and an iron core. The moving coil is wrapped around the plastic cylinder, which is connected to the mass in the mechanical system, and this group of components is spatially movable. The group of components that includes the iron core and two magnets is spatially fixed. Hence, the moving coil is movable relative to the iron core and the two magnets. 
$\omega_{0}, \bar{\phi}, R_{0}, \alpha$, and $\beta$. In the case of the UD model, (A.19) is applied to determine $\zeta, \omega_{0}, \bar{\phi}, R_{0}, L_{g}, \alpha$, and $\beta$. In the case of the NL model, (A.19), (35), (45), and (46) are applied to determine $\zeta, \omega_{0}, \bar{\phi}, R_{0}, \mathcal{P}_{g}, \mathcal{P}_{j}$, and $R_{j}$. The number of discretization, $n$, in (41) is determined based on the Hankel singular values of $1 /\left(Z_{c}^{\mathrm{NL}}(s)+R_{0}\right)$, which is obtained by removing the term representing the mechanical system, $\bar{\phi}^{2} s /\left(s^{2}+2 \zeta \omega_{0} s+\omega_{0}^{2}\right)$, from $Y_{e}(s)$.

The estimates are determined from the weighted nonlinear least-squares in (49) for each model. The two-step estimation procedure described in Section 3.2 is applied for each model, and the command, Isqnonlin, in the Optimization Toolbox of MATLAB [45] is used to carry out the numerical optimization in each step.

The measurements of $Y_{e}(\mathrm{j} \omega)$ in the range $10-10^{5}[\mathrm{rad} / \mathrm{s}]$ are tested. First, we determine $n$ as follows. The Hankel singular values of $1 /\left(Z_{c}^{\mathrm{NL}}(s)+R_{0}\right)$ are first computed for a large $n$; in this work, $n$ is selected as 7 . Figure 9 shows the computational result of the Hankel singular values, where the number of states corresponds to $n+1$. As shown in this figure, the Hankel singular values drastically drop between state $6(n=5)$ and state $7(n=6)$; we have selected $n=5$.

Tables 1, 2, 3, 4, and 5 list the estimated parameters. The estimated values of $\zeta, \omega_{0}, \bar{\phi}$, and $R_{0}$ for each model are similar. Figure 10 shows the Bode diagrams of the measured data and estimated models. The results for all of the model estimates match the measured data in the low-frequency range $\left(\omega<10^{2}\right)$ including at $50[\mathrm{rad} / \mathrm{s}]$; however, the results for the conventional models I, II, and III do not match the measured data in the mid- and high-frequency ranges $\left(10^{2}<\omega\right)$. This implies that the conventional models are insufficient to represent the effect 225 of the eddy currents in the high-frequency range. The results for the UD and NL models match the measured data in the high-frequency range $\left(10^{4}<\omega\right)$; in particular, the phase converges to -70 [deg], but the UD model results do not match the measured data in the mid-frequency range $\left(10^{2}<\omega<10^{4}\right)$. The physical reason for this is that the material of the iron core is supposed to ${ }_{230}$ be uniform and the magnetic flux leakage is neglected in the UD model. The 


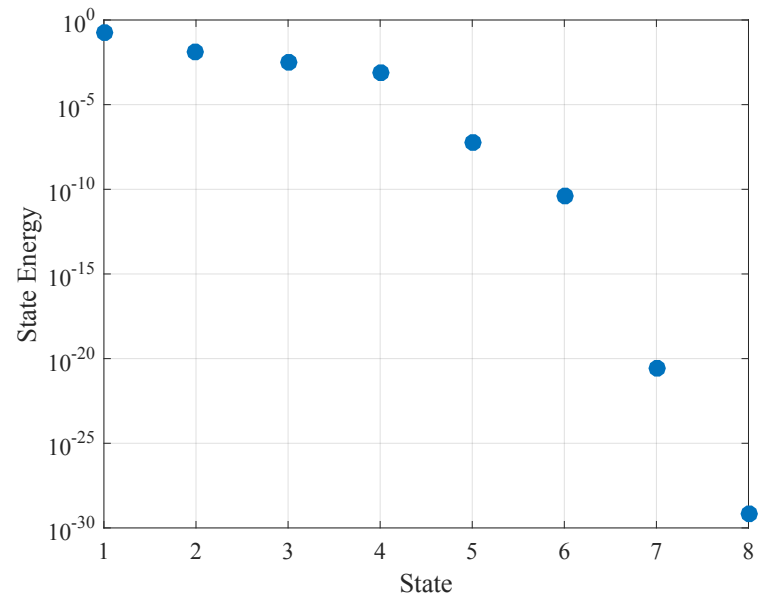

Figure 9: Hankel singular values of $1 /\left(Z_{c}^{\mathrm{NL}}(s)+R_{0}\right)$ when the number of state is $8(n=7)$. The number of state is selected as state $6(n=5)$ because the Hankel singular values drastically drop between state $6(n=5)$ and state $7(n=6)$.

computational reason for this is the difference in the number of parameters; only three parameters are included in $Z_{c}^{\mathrm{UD}}(s)$, whereas eleven parameters are included in $Z_{c}^{\mathrm{NL}}(s)$. Therefore, the NL model is more suitable than the conventional and UD models to represent the effect of the eddy currents. Modeling the actuator is obviously important for control, and the accurate modeling of the electromagnetic transducer in high-frequency ranges would be helpful in increasing the control bandwidth and improving the response of control systems for vibration and noise control, motion control, robotics, and other applications. We note that it is possible to estimate the values of $m, c, k$, and $\phi$ instead of $\zeta$, $240 \omega_{0}$, and $\bar{\phi}$. This optional estimation method is presented in Appendix B. 
Table 1: Estimated parameter values of the conventional model I.

\begin{tabular}{ccccc}
\hline$\zeta[-]$ & $\omega_{0}[\mathrm{rad} / \mathrm{s}]$ & $\bar{\phi}\left[\mathrm{N} / \mathrm{A} / \mathrm{kg}^{\frac{1}{2}}\right]$ & $R_{0}[\Omega]$ & $L_{0}[\mathrm{H}]$ \\
0.00847 & 50.8 & 1.66 & 2.57 & $1.65 \times 10^{-3}$ \\
\hline \hline
\end{tabular}

Table 2: Estimated parameter values of the conventional model II.

\begin{tabular}{cccccc}
\hline \hline$\zeta[-]$ & $\omega_{0}[\mathrm{rad} / \mathrm{s}]$ & $\bar{\phi}\left[\mathrm{N} / \mathrm{A} / \mathrm{kg}^{\frac{1}{2}}\right]$ & $R_{0}[\Omega]$ & $L_{0}[\mathrm{H}]$ & $R_{\mu}[\Omega]$ \\
0.00875 & 50.8 & 1.69 & 2.57 & $1.97 \times 10^{-3}$ & 295 \\
\hline \hline
\end{tabular}

Table 3: Estimated parameter values of the conventional model III.

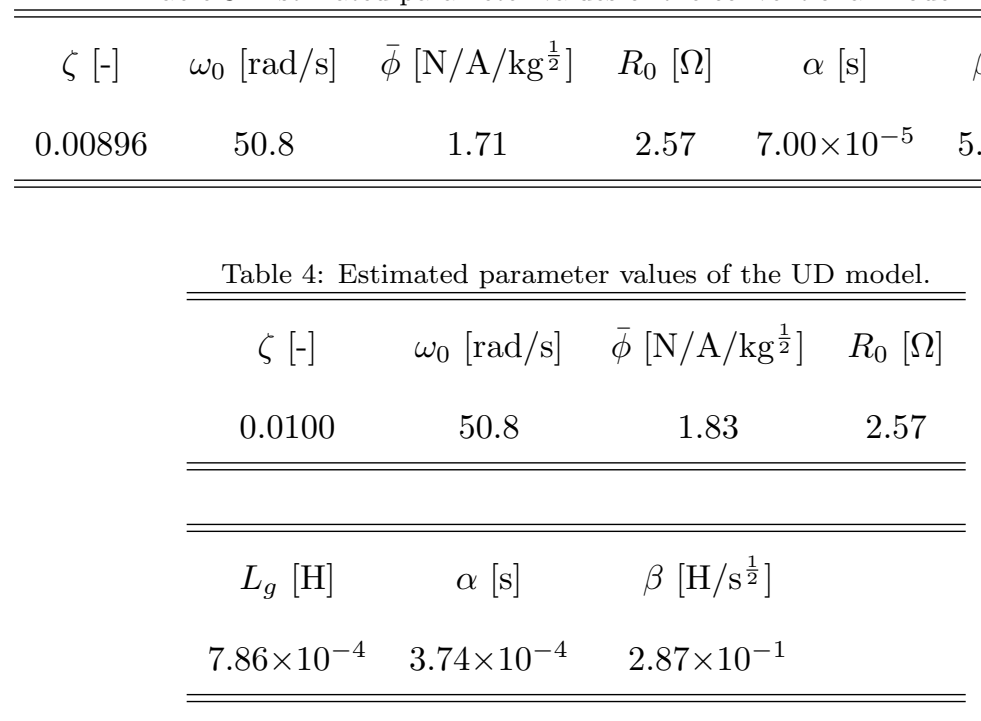

\section{Shunted vibration control experiments}

\subsection{Design of the $R C$-shunt circuit}

This section describes a shunted vibration control experiment using the RCshunt circuit [31], which is sensitive to the estimated parameters and is effective in evaluating the accuracy of the estimated models presented in the previous section. 
Table 5: Estimated parameter values of the NL model.

\begin{tabular}{ccccc}
\hline \hline \multicolumn{1}{c}{$\omega_{0}[\mathrm{rad} / \mathrm{s}]$} & $\bar{\phi}\left[\mathrm{N} / \mathrm{A} / \mathrm{kg}^{\frac{1}{2}}\right]$ & $R_{0}[\Omega]$ & $\mathcal{P}_{g}[\mathrm{H}]$ \\
0.0109 & 50.8 & 1.91 & 2.56 & $3.43 \times 10^{-9}$ \\
\hline \hline & & & \\
\hline \hline $\mathcal{P}_{1}[\mathrm{H}]$ & $\mathcal{P}_{2}[\mathrm{H}]$ & $\mathcal{P}_{3}[\mathrm{H}]$ & $\mathcal{P}_{4}[\mathrm{H}]$ & $\mathcal{P}_{5}[\mathrm{H}]$ \\
$4.09 \times 10^{-9}$ & $3.84 \times 10^{-9}$ & $3.73 \times 10^{-9}$ & $4.77 \times 10^{-9}$ & $4.28 \times 10^{-9}$ \\
\hline \hline & & & & \\
\hline \hline$R_{1}[\Omega]$ & $R_{2}[\Omega]$ & $R_{3}[\Omega]$ & $R_{4}[\Omega]$ & $R_{5}[\Omega]$ \\
$2.91 \times 10^{-6}$ & $1.43 \times 10^{-5}$ & $5.37 \times 10^{-5}$ & $1.90 \times 10^{-4}$ & $8.33 \times 10^{-4}$ \\
\hline \hline
\end{tabular}
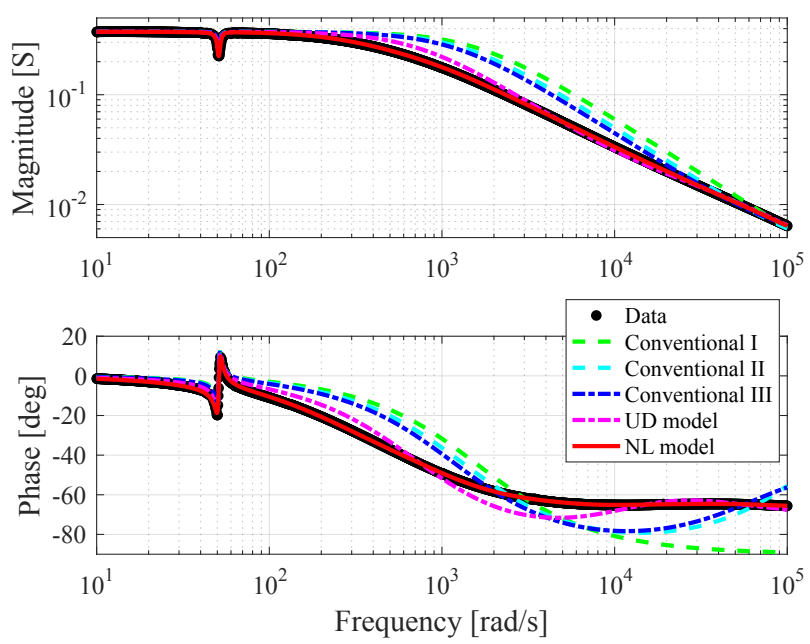

Figure 10: Bode admittance diagram of $Y_{e}^{\mathrm{I}}(\mathrm{j} \omega)$ (green broken line), $Y_{e}^{\mathrm{II}}(\mathrm{j} \omega)$ (cyan broken line), $Y_{e}^{\mathrm{III}}(\mathrm{j} \omega)$ (blue dashed line), $Y_{e}^{\mathrm{UD}}(\mathrm{j} \omega)$ (purple dashed line), $Y_{e}^{\mathrm{NL}}(\mathrm{j} \omega)$ (red solid line), and experimental data (black dots) in the range of $10^{1}-10^{5}[\mathrm{rad} / \mathrm{s}]$. The estimations obtained by all of the models match the measured data in the low-frequency range $\left(\omega<10^{2}\right)$; however, the results for the conventional models do not match the measured data in the mid- and high- frequency ranges $\left(10^{2}<\omega\right)$. The results obtained by the UD and NL models match the measured data in the high-frequency range $\left(10^{4}<\omega\right)$; however, the results obtained by the UD model do not match the measured data in the mid-frequency range $\left(10^{2}<\omega<10^{4}\right)$ 
By using (1)-(5), the transfer function from $f_{d}(t)$ to $x(t), G(s)$, is given by

$$
G(s):=\frac{\tilde{x}(s)}{\tilde{f}_{d}(s)}=\frac{1 / m}{s^{2}+2 \zeta \omega_{0} s+\omega_{0}^{2}+\frac{\bar{\phi}^{2} s}{Z_{c}(s)+R_{0}+Z_{s}(s)}},
$$

where $Z_{s}(s):=-\tilde{v}_{e}(s) / \tilde{i}_{e}(s)$ is the impedance of the shunt circuit and will be designed later. When the terminal of the electromagnetic transducer is open, that is, $i_{e}(t)=0, G(s)$ is denoted by $G_{\text {open }}(s)$ as follows:

$$
G_{\text {open }}(s)=\frac{1 / m}{s^{2}+2 \zeta \omega_{0} s+\omega_{0}^{2}} .
$$

When the RC-shunt circuit is connected to the terminal of the electromagnetic transducer, that is, $Z_{s}(s)=R_{s}+\frac{1}{C_{s} s}, G(s)$ is denoted by $G_{\mathrm{RC}}(s)$ as follows:

$$
G_{\mathrm{RC}}(s)=\frac{1 / m}{s^{2}+2 \zeta \omega_{0} s+\omega_{0}^{2}+\frac{\bar{\phi}^{2} s}{Z_{c}(s)+R_{0}+R_{s}+\frac{1}{C_{s} s}}},
$$

where $R_{s}[\Omega]$ is the resistance and $C_{s}[\mathrm{~F}]$ is the capacitance in the RC-shunt circuit [46].

For each estimated model, the problem of vibration control is formulated by selecting $R_{s}$ and $C_{s}$, which minimize the $H_{\infty}$ norm of $G_{\mathrm{RC}}(s),\left\|G_{\mathrm{RC}}\right\|_{\infty}$.

In the case of the conventional model I, the optimal values of $R_{s}$ and $C_{s}$ are given by $[22]$

$$
\begin{aligned}
R_{s}^{\mathrm{opt}} & =\frac{\bar{\phi}}{4}\left(\sqrt{6 L_{0}+\frac{\bar{\phi}}{\omega_{0}} \sqrt{2 L_{0}}}+\sqrt{6 L_{0}-\frac{\bar{\phi}}{\omega_{0}} \sqrt{2 L_{0}}}\right)-R_{0}, \\
C_{s}^{\mathrm{opt}} & =\frac{2}{2 \omega_{0}^{2} L_{0}-\bar{\phi}^{2}} .
\end{aligned}
$$

In the case of the UD or NL model, $R_{s}$ and $C_{s}$ are determined by numerical optimization. The initial values are given by (53) and (54). Figure 11 shows the contour map of $20 \log _{10}\left(\left\|G_{\mathrm{RC}}^{\mathrm{NL}}\right\|_{\infty} /\left\|G_{\text {open }}\right\|_{\infty}\right)$, which represents the gain drop of $G_{\mathrm{RC}}^{\mathrm{NL}}(s)$ from $G_{\text {open }}(s)$, and the red cross denotes the optimal values of $R_{s}$ and $C_{s}$. Note that $\left\|G_{\mathrm{RC}}^{\mathrm{NL}}\right\|_{\infty}$ is sensitive to $R_{s}$ and $C_{s}$, especially in the direction in which $R_{s}$ decreases from the optimal solution. Figure 12 shows the simulated frequency responses for $G_{\mathrm{RC}}^{\mathrm{NL}}(s)$ versus $G_{\text {open }}(s)$. The peak of $G_{\mathrm{RC}}^{\mathrm{NL}}(\mathrm{j} \omega)$ is separated into two peaks that are analogous to the dynamic vibration 
Table 6: Optimal designed parameters of the RC-shunt circuit from estimated results.

\begin{tabular}{cccccc}
\hline \hline & $\mathrm{I}$ & $\mathrm{II}$ & $\mathrm{III}$ & $\mathrm{UD}$ & $\mathrm{NL}$ \\
\hline$R_{s}^{\mathrm{opt}}[\Omega]$ & -2.49 & -2.48 & -2.47 & -2.43 & -2.40 \\
$C_{s}^{\mathrm{opt}}[\mathrm{F}]$ & 0.345 & 0.281 & 0.240 & 0.135 & 0.0814 \\
\hline \hline
\end{tabular}

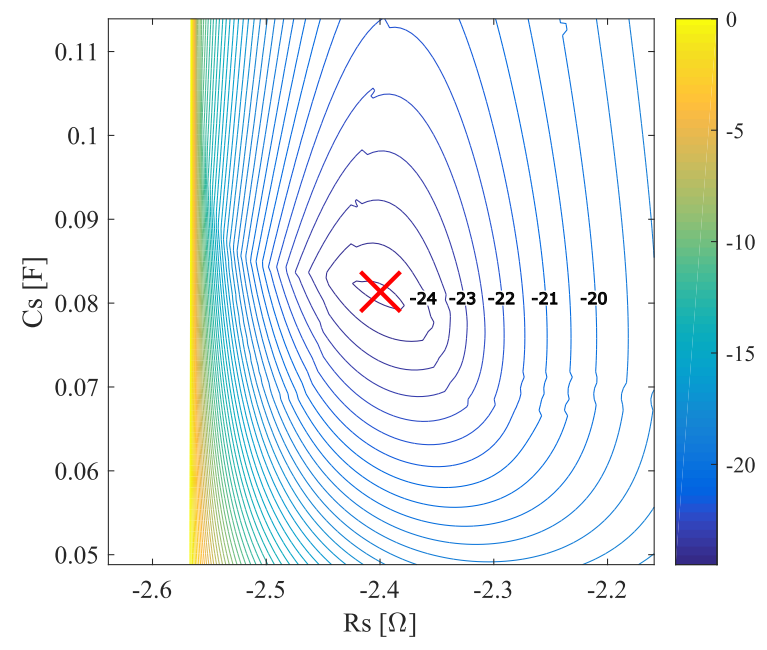

Figure 11: Contour map of $20 \log _{10}\left(\left\|G_{\mathrm{RC}}^{\mathrm{NL}}\right\|_{\infty} /\left\|G_{\mathrm{open}}\right\|_{\infty}\right)$ and the optimal solution obtained by the numerical optimization (red cross). The contour interval is $0.5[\mathrm{~dB}] .\left\|G_{\mathrm{RC}}^{\mathrm{NL}}\right\|_{\infty}$ is sensitive to $R_{s}$ and $C_{s}$, especially in the direction in which $R_{s}$ decreases from the optimal solution. The control performance of the RC-shunt circuit is shown to be sensitive to the selections of $R_{s}$ and $C_{s}$.

absorber, and the peak gain decreases to -24.7 [dB], where the peak gain of $G_{\text {open }}(s)$ is normalized to $0[\mathrm{~dB}]$. The results of the UD model are similar and are omitted here.

Table 6 lists the designed parameters of $R_{s}^{\mathrm{opt}}$ and $C_{s}^{\mathrm{opt}}$ for each model. The results of $R_{s}^{\mathrm{opt}}$ are similar but those of $C_{s}^{\mathrm{opt}}$ are different. As discussed in Section $3.2, \zeta, \omega_{0}, \bar{\phi}$, and $R_{0}$ are similar in each model, and the mismatch in the estimated frequency responses of $Y_{e}(s)$ in Fig. 10 is caused by $Z_{c}(s)$. Therefore, the design parameter of $C_{s}^{\text {opt }}$ for each model depends on the model of $Z_{c}(s)$, and $C_{s}^{\text {opt }}$ is different for each model. 


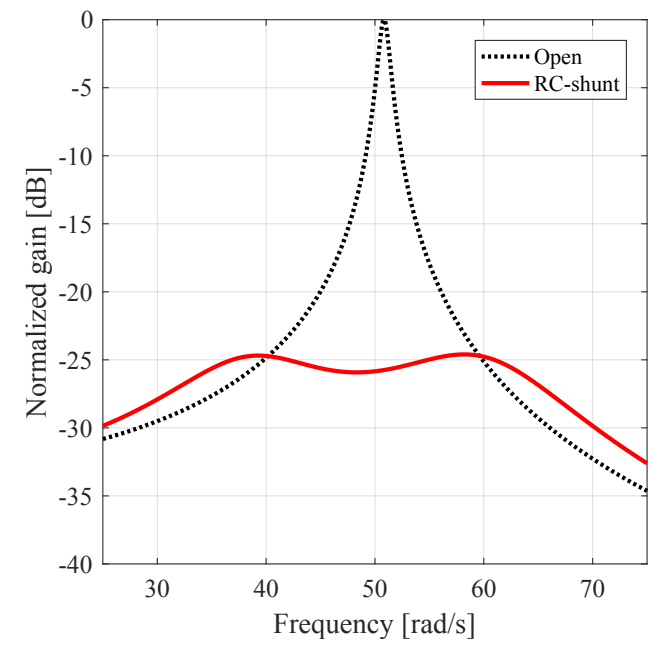

Figure 12: Simulated frequency response of the normalized gain from the disturbance force to the displacement, in which the black dotted line represents $G_{\text {open }}(\mathrm{j} \omega)$, whereas the red solid line represents $G_{\mathrm{RC}}^{\mathrm{NL}}(\mathrm{j} \omega)$. The peak gain of $G_{\mathrm{RC}}^{\mathrm{NL}}(\mathrm{j} \omega)$ drops by -24.7 [dB] compared with $G_{\text {open }}(s)$.

\subsection{Experimental method for vibration control}

Figure 13 shows the experimental setup [38]. The disturbance provided to the mass-spring-damper system is generated by the exciter, which is connected to the current amplifier and function generator. The displacement of the massspring-damper system is measured by the laser displacement meter (LB-080, KEYENCE). The amplitude of the applied voltage is $0.7[\mathrm{~V}]$, and the applied voltage, which is swept from $25[\mathrm{rad} / \mathrm{s}]$ to $75[\mathrm{rad} / \mathrm{s}]$, is applied for $80[\mathrm{~s}]$. The sampling frequency and the number of data points of the data logger are $200[\mathrm{~Hz}]$ and 16384 points, respectively. It should be noted that the laser displacement meter is only used for evaluating the vibration control performance and not for control. For averaging the frequency response, vibration control experiments are performed thrice for each model using the RC-shunt circuit. The frequency response, $G_{\mathrm{RC}}(\mathrm{j} \omega)$, is computed by dividing the cross power spectral density of $f_{d}(t)$ and $x(t)$ by the power spectral density of $f_{d}(t)$.

The shunt circuit is implemented using the synthetic impedance circuit [32, 


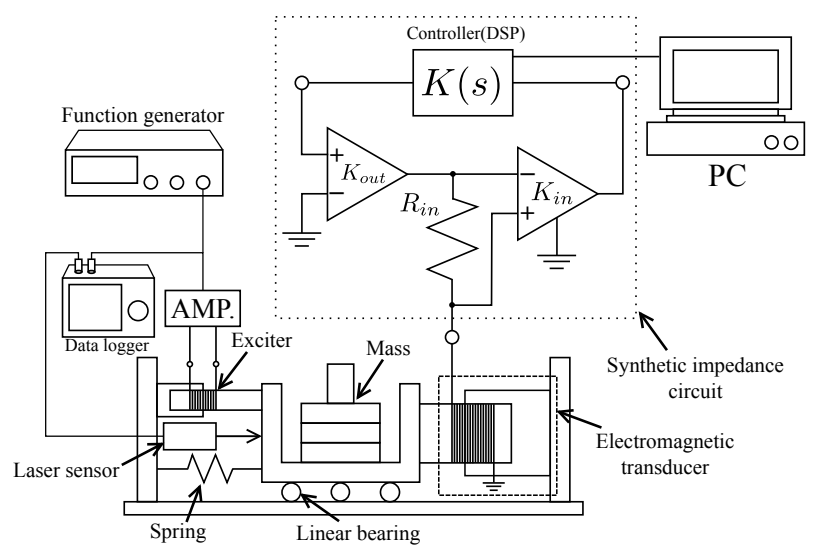

Figure 13: Schematic of the experimental setup used for shunt damping. A synthetic impedance circuit is coupled with the electromagnetic transducer for damping, and a current amplifier (AMP.) is coupled with the exciter for actuation [38].

Table 7: Parameters of the analog circuit.

\begin{tabular}{ccc}
\hline \hline$R_{\text {in }}[\Omega]$ & $K_{\text {in }[-]}$ & $K_{\text {out }}[-]$ \\
0.0996 & 99.23 & 0.9846 \\
\hline
\end{tabular}

46], which consists of the controller, $K(s)$, the instrumentation amplifier whose gain is $K_{\text {in }}$, the power amplifier whose gain is $K_{\text {out }}$, and the resistor, $R_{\text {in }}$, as shown in Fig. 13. The values of $K_{\text {in }}, K_{\text {out }}$, and $R_{\text {in }}$ are estimated by experiments and are summarized in Table 7 . In this implementation, $Z_{s}(s)$ is given by

$$
Z_{s}(s)=R_{\text {in }}+R_{\text {in }} K_{\text {in }} K_{\text {out }} K(s) .
$$

To implement the RC-shunt circuit, $K(s)$ is determined as follows:

$$
K(s)=\frac{1}{R_{\text {in }} K_{\text {in }} K_{\text {out }}}\left(R_{s}-R_{\text {in }}+\frac{1}{C_{s} s}\right) .
$$

A digital signal processor (DSP, s-BOX, MTT Corporation) and MATLAB/Simulink [47] are used to implement the controller, $K(s)$, discretized by the bilinear transformation. The sampling frequency of the DSP is $5000[\mathrm{~Hz}]$. 


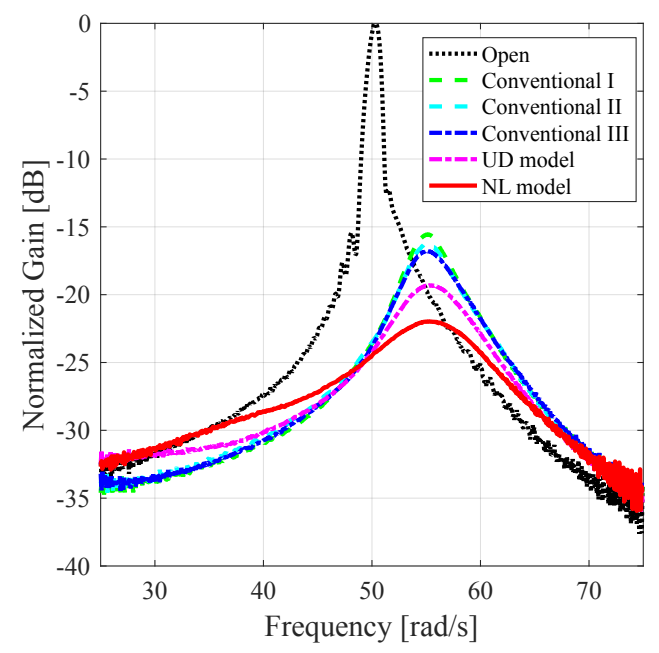

Figure 14: Frequency response of the normalized gain from the disturbance force to the displacement in the experiments (open terminals: black dotted line; conventional model I: green broken line; conventional model II: cyan broken line; conventional model III: blue dashed line; UD model: purple dashed line; NL model: red solid line). The NL model provides the most suitable modeling because its peak gain is the most attenuated. The UD model also provides more suitable modeling in comparison with the conventional models.

\subsection{Experimental results of vibration control}

Figure 14 shows the results of vibration control. The peak gains of the conventional models, I, II, and III, the UD model, and the NL model drop by -15.5 , $-16.3,-16.8,-19.3$, and $-22.0[\mathrm{~dB}]$, respectively. The control performance is sensitive to the designed parameters $R_{s}$ and $C_{s}$, as discussed in Section 5.1. It is obvious that the quality of the model has an effect on the control performance. Therefore, the NL model is the most suitable one, and the UD model is also better than the conventional models.

\section{Conclusions}

In this paper, a method is presented for simultaneously estimating all the parameters of an electromagnetic transducer by only measuring the frequency response of the admittance; the method does not require any additional sensors. This paper also discusses the importance of the effect of eddy currents in 
impedance modeling. Two novel models have been presented; the UD model is the impedance model that considers spatially uniform parameters and spatially distributed eddy currents, whereas the NL model is the impedance model that considers spatially nonuniform parameters and spatially lumped eddy currents. Both the proposed models improve the accuracy of the frequency response of the admittance in the mid- and high-frequency ranges compared to the conventional models. The UD and NL models play complementary roles; the tradeoff is between a smaller number of parameters in the former and a higher estimation accuracy in the latter. The results of vibration control experiments show that the accurate estimate provided by the proposed models improves the control performance of shunt damping, which is sensitive to the parameters of self-induction.

\section{Acknowledgment}

This work was partially supported by the JSPS KAKENHI Grant Numbers 26420174, 15K06136.

\section{Appendix A. Selection of an initial estimate}

In the same way as [38], this section presents a method for selecting an initial estimate of the nonlinear least-squares in (49). By rearranging the parameters $\boldsymbol{\theta}_{\text {nls }}$ in a new set of parameters $\boldsymbol{\theta}_{\text {ini }}$, (47) can be rewritten in the fractional form as

$$
Y_{e}(s)=\frac{g_{\mathrm{num}}\left(s ; \boldsymbol{\theta}_{\mathrm{ini}}\right)}{g_{\mathrm{den}}\left(s ; \boldsymbol{\theta}_{\mathrm{ini}}\right)}
$$

where $g_{\text {num }}\left(s ; \boldsymbol{\theta}_{\text {ini }}\right)$ is a numerator polynomial of $s$ and is affine to $\boldsymbol{\theta}_{\text {ini }}$, and $g_{\text {den }}\left(s ; \boldsymbol{\theta}_{\text {ini }}\right)$ is a denominator polynomial of $s$ and is also affine to $\boldsymbol{\theta}_{\text {ini }}$. By multiplying the numerator polynomial, $g_{\text {num }}\left(s ; \boldsymbol{\theta}_{\text {ini }}\right)$, with the cost function in (49), the parameter estimation problem is reformulated by the weighted linear 
least-squares method in the frequency domain.

$$
\min _{\boldsymbol{\theta}_{\text {ini }}} \sum_{k=1}^{N_{s}}\left|\left(\frac{g_{\text {num }}\left(\mathrm{j} \omega_{s}[k] ; \boldsymbol{\theta}_{\text {ini }}\right)}{Y_{s}\left(\mathrm{j} \omega_{s}[k]\right)}-g_{\text {den }}\left(\mathrm{j} \omega_{s}[k] ; \boldsymbol{\theta}_{\text {ini }}\right)\right) W_{\text {ini }}\left(\mathrm{j} \omega_{s}[k]\right)\right|^{2},
$$

where $W_{\text {ini }}\left(\mathrm{j} \omega_{s}[k]\right)$ is the weighting function given by $W_{\text {ini }}\left(j \omega_{s}[k]\right):=1 / \mathrm{j} \omega_{s}[k]$. The weighted linear least-squares in (A.2) can be rewritten in the matrix form as

$$
\min _{\boldsymbol{\theta}_{\text {ini }}}\left\|\boldsymbol{A} \boldsymbol{\theta}_{\text {ini }}-\boldsymbol{b}\right\|^{2}
$$

The optimal solution of (A.3) is given by

$$
\boldsymbol{\theta}_{\text {iniopt }}=\left(\boldsymbol{A}^{*} \boldsymbol{A}+\overline{\boldsymbol{A}^{*} \boldsymbol{A}}\right)^{-1}\left(\boldsymbol{A}^{*} \boldsymbol{b}+\overline{\boldsymbol{A}^{*} \boldsymbol{b}}\right)
$$

where $\boldsymbol{A}^{*}$ is the complex conjugate transpose of $\boldsymbol{A}$ and $\overline{\boldsymbol{A}}$ is the complex conjugate of $\boldsymbol{A}$.

The initial estimate and its detailed derivation for the conventional model I given in (6) have been described in [38]. In the same manner, the initial estimates and their detailed derivations for the other impedance models given in (7), (22), (21), and (41) are described in the following subsections. We note

that it is not straightforward to determine $\boldsymbol{\theta}_{\text {nls }}$ from the optimized $\boldsymbol{\theta}_{\text {iniopt }}$ in (A.4) for the following reasons: (i) $\boldsymbol{\theta}_{\text {ini }}$ is nonlinearly coupled with $\boldsymbol{\theta}_{\text {nls }}$, (ii) $\boldsymbol{\theta}_{\text {ini }}$ is generically redundant to determine $\boldsymbol{\theta}_{\mathrm{nls}}$, and (iii) the conventional model III and the UD model are not rational functions. Therefore, approximations are carried out to determine $\boldsymbol{\theta}_{\text {nls }}$ from $\boldsymbol{\theta}_{\text {iniopt }}$ in each model.

\section{Appendix A.1. Conventional model II}

In the case of the conventional model II given in $(7), Y_{e}^{\mathrm{II}}(s)$ is represented in the form of (A.1), and then, $\boldsymbol{\theta}_{\text {ini }}^{\mathrm{II}}$ is obtained as follows:

$$
\begin{aligned}
\boldsymbol{\theta}_{\text {ini }}^{\mathrm{II}}:= & {\left[\frac{L_{0}}{R_{\mu} \omega_{0}^{2}}, \frac{1}{\omega_{0}^{2}}\left(1+\frac{2 L_{0} \zeta \omega_{0}}{R_{\mu}}\right), \frac{L_{0}}{R_{\mu}}+\frac{2 \zeta}{\omega_{0}}, \frac{L_{0}}{\omega_{0}^{2}}\left(\frac{R_{0}}{R_{\mu}}+1\right),\right.} \\
& \frac{R_{0}}{\omega_{0}^{2}}+\frac{L_{0} \bar{\phi}^{2}}{R_{\mu} \omega_{0}^{2}}+\frac{2 L_{0} \zeta}{\omega_{0}}\left(\frac{R_{0}}{R_{\mu}}+1\right) \\
& \left.L_{0}\left(\frac{R_{0}}{R_{\mu}}+1\right)+\frac{\bar{\phi}^{2}}{\omega_{0}^{2}}+\frac{2 R_{0} \zeta}{\omega_{0}}, R_{0}\right]^{\mathrm{T}} .
\end{aligned}
$$


The $k$-th row components of $\boldsymbol{A}^{\mathrm{II}}$ and $\boldsymbol{b}^{\mathrm{II}}, A_{k}^{\mathrm{II}}$ and $b_{k}^{\mathrm{II}}, k=1, \cdots, N_{s}$, are given by

$$
\begin{aligned}
A_{k}^{\mathrm{II}} & :=\left[\frac{\left(\mathrm{j} \omega_{s}[k]\right)^{2}}{Y_{s}\left(\mathrm{j} \omega_{s}[k]\right)}, \frac{\mathrm{j} \omega_{s}[k]}{Y_{s}\left(\mathrm{j} \omega_{s}[k]\right)}, \frac{1}{Y_{s}\left(\mathrm{j} \omega_{s}[k]\right)},\right. \\
& \left.-\left(\mathrm{j} \omega_{s}[k]\right)^{2},-\mathrm{j} \omega_{s}[k],-1,-\frac{1}{\mathrm{j} \omega_{s}[k]}\right], \\
b_{k}^{\mathrm{II}} & :=-\frac{1}{\mathrm{j} \omega_{s}[k] Y_{s}\left(\mathrm{j} \omega_{s}[k]\right)} .
\end{aligned}
$$

By substituting (A.6) and (A.7) into (A.4), $\boldsymbol{\theta}_{\text {iniopt }}^{\mathrm{II}}$ is determined. By evaluating the numerical orders, we have $L_{0} \ll 1, \zeta \ll 1, R_{\mu} \gg 1$, and $\omega_{0}>1$ in practice, and therefore, $2 L_{0} \zeta \omega_{0} / R_{\mu} \ll 1$, i.e., the second term in the second component of (A.5) can be neglected. By equating (A.4) and (A.5), the initial estimates of the nonlinear least-squares method for the conventional model II, $\zeta, \omega_{0}, \bar{\phi}, R_{0}$, $L_{0}$, and $R_{\mu}$, are selected as follows:

$$
\begin{aligned}
\zeta & =\frac{1}{2 \sqrt{\theta_{2}}}\left(\theta_{3}-\frac{\theta_{1}}{\theta_{2}}\right), \quad \omega_{0}=\frac{1}{\sqrt{\theta_{2}}}, \\
\bar{\phi} & =\sqrt{\frac{\theta_{6}-\theta_{3} \theta_{7}}{\theta_{2}}+\frac{\theta_{1} \theta_{7}-\theta_{4}}{\theta_{2}^{2}}, \quad R_{0}=\theta_{7},} \\
L_{0} & =\frac{\theta_{4}-\theta_{1} \theta_{7}}{\theta_{2}}, \quad R_{\mu}=\frac{\theta_{4}-\theta_{1} \theta_{7}}{\theta_{1}} .
\end{aligned}
$$

where $\theta_{i}, i=1, \cdots, 7$, are the components of $\boldsymbol{\theta}_{\text {iniopt }}^{\mathrm{II}}$.

\section{Appendix A.2. Conventional model III}

In the case of the conventional model III given in (22), it is difficult to represent $Y_{e}^{\mathrm{III}}(s)$ in the form of (A.1), and this term is therefore approximated. The Maclaurin expansion of $J_{\nu}(z)$ is given by

$$
J_{\nu}(z)=\left(\frac{z}{2}\right)^{\nu} \sum_{k=0}^{\infty} \frac{\left(-z^{2} / 4\right)^{k}}{k ! \Gamma(\nu+k+1)}
$$

where $\Gamma(z)$ is the gamma function[48]. Equation (A.9) is substituted into $Z_{c}^{\mathrm{III}}(s)$ in (22). By taking the first-order components of the numerator and the denominator of $Z_{c}^{\mathrm{III}}(s)$, the first-order approximation of $Z_{c}^{\mathrm{III}}(s)$ is given by

$$
Z_{c}^{\mathrm{III}}(s) \approx \sqrt{\alpha} \beta s \frac{\alpha s+8}{4 \alpha s+16} .
$$


$Y_{e}^{\mathrm{III}}(s)$ is approximated by substituting (A.10) into (47). The approximated $Y_{e}^{\mathrm{III}}(s)$ is rewritten in the form of (A.1), and then, $\boldsymbol{\theta}_{\mathrm{ini}}^{\mathrm{III}}$ is obtained as follows:

$$
\begin{aligned}
\boldsymbol{\theta}_{\mathrm{ini}}^{\mathrm{III}} & :=\left[\frac{\alpha}{4 \omega_{0}^{2}}, \frac{1}{\omega_{0}^{2}}\left(1+\frac{\alpha \zeta \omega_{0}}{2}\right), \frac{2 \zeta}{\omega_{0}}+\frac{\alpha}{4}, \frac{\alpha^{3 / 2} \beta}{16 \omega_{0}^{2}},\right. \\
& \alpha\left(\frac{R_{0}}{4 \omega_{0}^{2}}+\frac{\sqrt{\alpha} \beta}{8 \omega_{0}} \zeta\right)+\frac{\sqrt{\alpha} \beta}{2 \omega_{0}^{2}}, \\
& \alpha\left(\frac{\bar{\phi}^{2}}{4 \omega_{0}^{2}}+\frac{R_{0} \zeta}{2 \omega_{0}}\right)+\sqrt{\alpha} \beta\left(\frac{\zeta}{\omega_{0}}+\frac{\alpha}{16}\right)+\frac{R_{0}}{\omega_{0}^{2}} \\
& \left.\frac{\sqrt{\alpha} \beta}{2}+\frac{R_{0}}{4}\left(\alpha+\frac{8 \zeta}{\omega_{0}}\right)+\frac{\bar{\phi}^{2}}{\omega_{0}^{2}}, R_{0}\right]^{\mathrm{T}} .
\end{aligned}
$$

The $k$-th row components of $\boldsymbol{A}^{\mathrm{III}}$ and $\boldsymbol{b}^{\mathrm{III}}, A_{k}^{\mathrm{III}}$ and $b_{k}^{\mathrm{III}}, k=1, \cdots, N_{s}$, are given by

$$
\begin{aligned}
A_{k}^{\mathrm{III}} & :=\left[\frac{\left(\mathrm{j} \omega_{s}[k]\right)^{2}}{Y_{s}\left(\mathrm{j} \omega_{s}[k]\right)}, \frac{\mathrm{j} \omega_{s}[k]}{Y_{s}\left(\mathrm{j} \omega_{s}[k]\right)}, \frac{1}{Y_{s}\left(\mathrm{j} \omega_{s}[k]\right)},-\left(\mathrm{j} \omega_{s}[k]\right)^{3},\right. \\
& \left.-\left(\mathrm{j} \omega_{s}[k]\right)^{2},-\mathrm{j} \omega_{s}[k],-1,-\frac{1}{\mathrm{j} \omega_{s}[k]}\right], \\
b_{k}^{\mathrm{III}} & :=-\frac{1}{\mathrm{j} \omega_{s}[k] Y_{s}\left(\mathrm{j} \omega_{s}[k]\right)} .
\end{aligned}
$$

By substituting (A.12) and (A.13) into (A.4), $\boldsymbol{\theta}_{\text {iniopt }}^{\mathrm{III}}$ is determined. By evaluating the numerical orders, we have $\alpha \ll 1, \zeta \ll 1$, and $\omega_{0}>1$ in practice, and therefore, $\alpha \zeta \omega_{0} / 2 \ll 1$, i.e., the second term in the second component of (A.11) can be neglected. By equating (A.4) and (A.11), the initial estimates of the nonlinear least-squares method for the conventional model III, $\zeta, \omega_{0}, \bar{\phi}, R_{0}$, $\alpha$, and $\beta$, are selected as follows:

$$
\begin{aligned}
\zeta & =\frac{1}{2 \sqrt{\theta_{2}}}\left(\theta_{3}-\frac{\theta_{1}}{\theta_{2}}\right), \quad \omega_{0}=\frac{1}{\sqrt{\theta_{2}}}, \\
\bar{\phi} & =\sqrt{\frac{1}{\theta_{1}}\left(\theta_{6}+\frac{\theta_{4}}{\theta_{2}}-\theta_{2} \theta_{8}\right)+\frac{\theta_{1} \theta_{8}}{\theta_{2}^{2}}-2 \frac{\theta_{3} \theta_{4}}{\theta_{1}^{2}}-\frac{\theta_{3} \theta_{8}}{\theta_{2}}, \quad R_{0}=\theta_{8},} \\
\alpha & =\frac{4 \theta_{1}}{\theta_{2}}, \quad \beta=2 \frac{\theta_{4}}{\theta_{1}} \sqrt{\frac{\theta_{2}}{\theta_{1}}} .
\end{aligned}
$$

where $\theta_{i}, i=1, \cdots, 8$, are the components of $\boldsymbol{\theta}_{\text {iniopt }}^{\text {III }}$.

\section{Appendix A.3. UD model}

In the case of the UD model given in (21), it is difficult to represent $Y_{e}^{\mathrm{UD}}(s)$ in the form of (A.1), and therefore, approximation is carried out. Equation 
(A.9) is substituted into $Z_{c}^{\mathrm{UD}}(s)$ in (21). By taking the first-order components of the numerator and the denominator of $Z_{c}^{\mathrm{UD}}(s)$, the first-order approximation of $Z_{c}^{\mathrm{UD}}(s)$ is given by

$$
Z_{c}^{\mathrm{UD}}(s) \approx L_{g} s+\sqrt{\alpha} \beta s \frac{\alpha s+8}{4 \alpha s+16} .
$$

By substituting (A.15) into (47), $Y_{e}^{\mathrm{UD}}(s)$ is rewritten in the form of (A.1), and then, $\boldsymbol{\theta}_{\text {ini }}^{\mathrm{UD}}$ is obtained as follows:

$$
\begin{aligned}
\boldsymbol{\theta}_{\mathrm{ini}}^{\mathrm{UD}} & :=\left[\frac{\alpha}{4 \omega_{0}^{2}}, \frac{1}{\omega_{0}^{2}}\left(1+\frac{\alpha \zeta \omega_{0}}{2}\right), \frac{2 \zeta}{\omega_{0}}+\frac{\alpha}{4}, \frac{4 L_{g} \alpha+\alpha^{3 / 2} \beta}{16 \omega_{0}^{2}},\right. \\
& \alpha\left(\frac{R_{0}}{4 \omega_{0}^{2}}+\frac{4 L_{g}+\sqrt{\alpha} \beta}{8 \omega_{0}} \zeta\right)+\frac{2 L_{g}+\sqrt{\alpha} \beta}{2 \omega_{0}^{2}}, \\
& \alpha\left(\frac{\bar{\phi}^{2}}{\omega_{0}^{2}}+\frac{R_{0} \zeta}{2 \omega_{0}}+\frac{L_{g}}{4}\right)+\sqrt{\alpha} \beta\left(\frac{\zeta}{\omega_{0}}+\frac{\alpha}{16}\right)+\frac{R_{0}}{\omega_{0}^{2}}+\frac{2 L_{g} \zeta}{\omega_{0}}, \\
& \left.L_{g}+\frac{\sqrt{\alpha} \beta}{2}+\frac{R_{0}}{4}\left(\alpha+\frac{8 \zeta}{\omega_{0}}\right)+\frac{\bar{\phi}^{2}}{\omega_{0}^{2}}, R_{0}\right]^{\mathrm{T}} .
\end{aligned}
$$

The $k$-th row components of $\boldsymbol{A}^{\mathrm{UD}}$ and $\boldsymbol{b}^{\mathrm{UD}}, A_{k}^{\mathrm{UD}}$ and $b_{k}^{\mathrm{UD}}, k=1, \cdots, N_{s}$, are given by

$$
\begin{aligned}
A_{k}^{\mathrm{UD}} & :=\left[\frac{\left(\mathrm{j} \omega_{s}[k]\right)^{2}}{Y_{s}\left(\mathrm{j} \omega_{s}[k]\right)}, \frac{\mathrm{j} \omega_{s}[k]}{Y_{s}\left(\mathrm{j} \omega_{s}[k]\right)}, \frac{1}{Y_{s}\left(\mathrm{j} \omega_{s}[k]\right)},-\left(\mathrm{j} \omega_{s}[k]\right)^{3},\right. \\
& \left.-\left(\mathrm{j} \omega_{s}[k]\right)^{2},-\mathrm{j} \omega_{s}[k],-1,-\frac{1}{\mathrm{j} \omega_{s}[k]}\right], \\
b_{k}^{\mathrm{UD}} & :=-\frac{1}{\mathrm{j} \omega_{s}[k] Y_{s}\left(\mathrm{j} \omega_{s}[k]\right)} .
\end{aligned}
$$

By substituting (A.17) and (A.18) into (A.4), $\boldsymbol{\theta}_{\text {iniopt }}^{\text {UD }}$ is determined. By evaluating the numerical orders, we have $\alpha \ll 1, \zeta \ll 1$, and $\omega_{0}>1$ in practice, and therefore, $\alpha \zeta \omega_{0} / 2 \ll 1$, i.e., the second term in the second component of (A.16) can be neglected. By equating (A.4) and (A.16), the initial estimates of the nonlinear least-squares method for the UD model, $\zeta, \omega_{0}, \bar{\phi}, R_{0}, L_{g}$, $\alpha$, and 
$\beta$, are selected as follows:

$$
\begin{aligned}
\zeta & =\frac{1}{2 \sqrt{\theta_{2}}}\left(\theta_{3}-\frac{\theta_{1}}{\theta_{2}}\right), \quad \omega_{0}=\frac{1}{\sqrt{\theta_{2}}}, \\
\bar{\phi} & =\sqrt{\frac{\theta_{7}-\theta_{3} \theta_{8}}{\theta_{2}}+\frac{\theta_{1} \theta_{8}-\theta_{5}}{\theta_{2}^{2}}+\frac{\theta_{4}}{\theta_{2}^{3}}\left(\theta_{3}-\frac{\theta_{1}}{\theta_{2}}\right)}, \quad R_{0}=\theta_{8}, \\
L_{g} & =\theta_{1}\left(\frac{\theta_{8}}{\theta_{2}}-\frac{\theta_{4}}{\theta_{2}^{3}}\right)+\left(\frac{2}{\theta_{1}}+\frac{\theta_{3}}{\theta_{2}^{2}}\right) \theta_{4}-\frac{\theta_{5}}{\theta_{2}}, \quad \alpha=\frac{4 \theta_{1}}{\theta_{2}} \\
\beta & =2 \sqrt{\frac{\theta_{1}}{\theta_{2}}}\left\{\frac{\theta_{5}}{\theta_{1}}+\frac{\theta_{4}}{\theta_{2}^{2}}-\theta_{8}-\frac{\theta_{4}}{\theta_{1}}\left(\frac{\theta_{3}}{\theta_{2}}+\frac{\theta_{2}}{\theta_{1}}\right)\right\} .
\end{aligned}
$$

where $\theta_{i}, i=1, \cdots, 8$, are the components of $\boldsymbol{\theta}_{\text {iniopt }}^{\mathrm{UD}}$.

\section{Appendix A.4. NL model}

In the case of the NL model given in (41), the initial estimates of the nonlinear least-squares method for the NL model are determined by those of the UD model. Specifically, the parameters $\zeta, \omega_{0}, \bar{\phi}$, and $R_{0}$ are the same as those in (A.19). The parameters $\mathcal{P}_{g}, \mathcal{P}_{j}$, and $R_{j}$ are determined by substituting the parameters $L_{g}, \alpha$, and $\beta$ in (A.19) into (35), (45), and (46).

\section{Appendix B. Mechanical parameter estimation using additional mass}

This section presents a method for estimating the values of $m, c, k$, and $\phi$ instead of $\zeta, \omega_{0}$, and $\bar{\phi}$. To this end, the additional mass, $\Delta m$, is added to

${ }^{40}$ the mass, $m$, and then, the frequency response of the admittance, $Y_{e}(\mathrm{j} \omega)$, is measured again. Then, $\zeta, \omega_{0}$, and $\bar{\phi}$ are changed to $\zeta^{\prime}:=c / \sqrt{4(m+\Delta m) k}$, $\omega_{0}^{\prime}:=\sqrt{k /(m+\Delta m)}$, and $\bar{\phi}^{\prime}:=\phi / \sqrt{m+\Delta m}$, respectively. The values of $m$, $c, k$, and $\phi$ can be calculated by using $\zeta, \omega_{0}, \bar{\phi}, \omega_{0}^{\prime}$, and $\Delta m$.

An additional measurement of $Y_{e}(\mathrm{j} \omega)$ is performed by adding $\Delta m=-0.645$ $[\mathrm{kg}]$, that is, by removing $0.645[\mathrm{~kg}]$ from the mass. Table B.8 lists the estimated parameters of the NL model including the values of $\zeta^{\prime}, \omega_{0}^{\prime}$, and $\bar{\phi}^{\prime}$. Table B.9 lists the estimated values of $m, c, k$, and $\phi$, which seem to be reliable because the estimated values of $R_{0}, \mathcal{P}_{g}, \mathcal{P}_{j}$, and $R_{j}$, which are not influenced by changes in $m$, are quite similar to the ones listed in Tables 5 and B.8. 
Table B.8: Estimated parameter values when $\Delta \mathrm{m}(=0.645 \mathrm{~kg})$ is removed.

\begin{tabular}{ccccc}
\hline \hline$\zeta^{\prime}[-]$ & $\omega_{0}^{\prime}[\mathrm{rad} / \mathrm{s}]$ & $\bar{\phi}^{\prime}\left[\mathrm{N} / \mathrm{A} / \mathrm{kg}^{\frac{1}{2}}\right]$ & $R_{0}[\Omega]$ & $\mathcal{P}_{g}[\mathrm{H}]$ \\
0.00927 & 64.2 & 2.26 & 2.58 & $3.43 \times 10^{-9}$ \\
\hline \hline & & & \\
\hline $\mathcal{P}_{1}[\mathrm{H}]$ & $\mathcal{P}_{2}[\mathrm{H}]$ & $\mathcal{P}_{3}[\mathrm{H}]$ & $\mathcal{P}_{4}[\mathrm{H}]$ & $\mathcal{P}_{5}[\mathrm{H}]$ \\
$4.16 \times 10^{-9}$ & $3.84 \times 10^{-9}$ & $3.73 \times 10^{-9}$ & $4.78 \times 10^{-9}$ & $4.28 \times 10^{-9}$ \\
\hline \hline & & & & \\
\hline \hline$R_{1}[\Omega]$ & $R_{2}[\Omega]$ & $R_{3}[\Omega]$ & $R_{4}[\Omega]$ & $R_{5}[\Omega]$ \\
$2.90 \times 10^{-6}$ & $1.43 \times 10^{-5}$ & $5.34 \times 10^{-5}$ & $1.89 \times 10^{-4}$ & $8.30 \times 10^{-4}$ \\
\hline \hline
\end{tabular}

Table B.9: Estimated parameter values of the mechanical model and electromechanical coupling coefficient.

\begin{tabular}{cccc}
\hline \hline$m[\mathrm{~kg}]$ & $c[\mathrm{Ns} / \mathrm{m}]$ & $k[\mathrm{~N} / \mathrm{m}]$ & $\phi[\mathrm{N} / \mathrm{A}]$ \\
1.73 & 1.92 & $4.47 \times 10^{3}$ & 2.51 \\
\hline \hline
\end{tabular}

\section{References}

[1] Boldea I, Nasar SA. Linear electric actuators and generators. IEEE Trans Energy Convers 1999;14(3):712-7. doi:10.1109/60.790940.

[2] Howe D. Magnetic actuators. Sensors Actuators A Phys 2000;81(1-3):26874. doi:10.1016/S0924-4247 (99)00174-0.

[3] Hanson B, Levesley M. Self-sensing applications for electromagnetic actuators. Sensors Actuators A Phys 2004;116(2):345-51. doi:10.1016/j.sna.2004.05.003.

[4] Yin W, Peyton AJ, Dickinson SJ. Simultaneous measurement of distance and thickness of a thin metal plate with an electromagnetic sensor us- 
ing a simplified model. IEEE Trans Instrum Meas 2004;53(4):1335-8. doi:10.1109/TIM. 2004.830585.

[5] Chen MY, Tzeng HW, Hung SK. A new mechanism design of electromagnetic actuator for a micro-positioner. ISA Trans 2007;46(1):41-8. doi:10.1016/j. isatra.2006.06.003.

[6] Song S, Li Z, Yu H, Ren H. Shape reconstruction for wire-driven flexible robots based on Bézier curve and electromagnetic positioning. Mechatronics 2015;29:28-35. doi:10.1016/j.mechatronics .2015.05.003.

[7] Rashedin R, Meydan T, Borza F. Electromagnetic micro-actuator array for loudspeaker application. Sensors Actuators A Phys 2006;129(1-2):118-20. doi:10.1016/j.sna.2005.11.050.

[8] Thiele N. Loudspeakers in vented boxes: Part 1. J Audio Eng Soc 1971;19(5):382-92.

[9] Sturtzer E, Pillonnet G, Lemarquand G, Abouchi N. Comparison between voltage and current driving methods of a micro-speaker. Applied Acoustics 2012;73(11):1087-98. doi:10.1016/j . apacoust. 2012 .05.008.

[10] Lammeraner J, Štafl M. Eddy currents. London: Iliffe; 1966. ISBN 0592050165 .

[11] Vanderkooy J. A model of loudspeaker driver impedance incorporating eddy currents in the pole structure. J Audio Eng Soc 1989;37(3):119-28.

380 [12] Shaw J. Active Vibration Isolation by Adaptive Control. J Vib Control 2001;7(1):19-31. doi:10.1177/107754630100700102.

[13] Eyabi P, Washington G. Design and control of an electromagnetic valve actuator. Mechatronics 2006;16(3-4):159-75. doi:10.1016/j.mechatronics.2005.11.008. 
[16] Kawamoto Y, Suda Y, Inoue H, Kondo T. Modeling of Electromagnetic Damper for Automobile Suspension. J Syst Des Dyn 2007;1(3):524-35. doi:10.1299/jsdd.1.524.

[17] van der Sande TPJ, Gysen BLJ, Besselink IJM, Paulides JJH, Lomonova

[19] Wang DA, Chiu CY, Pham HT. Electromagnetic energy harvesting from vibrations induced by Kármán vortex street. Mechatronics 2012;22(6):74656. doi:10.1016/j.mechatronics.2012.03.005.

[20] Zuo L, Scully B, Shestani J, Zhou Y. Design and characterization of an elec-

[14] Rossini L, Onillon E, Chételat O, Perriard Y. Closed-loop magnetic bearing and angular velocity control of a reaction sphere actuator. Mechatronics 2015;30:214-24. doi:10.1016/j .mechatronics .2015 .07.003.

[15] Choi C, Park K. Self-sensing magnetic levitation using a LC resonant circuit. Sensors Actuators A Phys 1999;72(2):169-77. EA, Nijmeijer H. Robust control of an electromagnetic active suspension system: Simulations and measurements. Mechatronics 2013;23(2):204-12. doi:10.1016/j.mechatronics.2012.07.002.

[18] Su X, Yang X, Shi P, Wu L. Fuzzy control of nonlinear electromagnetic suspension systems. Mechatronics 2014;24(4):328-35. doi:10.1016/j.mechatronics. 2013.08.002.

tromagnetic energy harvester for vehicle suspensions. Smart Mater Struct 2010;19(4):045003. doi:10 .1088/0964-1726/19/4/045003.

[21] Dayal R, Dwari S, Parsa L. Design and implementation of a direct ACDC boost converter for low-voltage energy harvesting. IEEE Trans Ind Electron 2011;58(6):2387-96. doi:10.1109/TIE. 2010.2069074.

[22] Inoue T, Ishida Y, Sumi M. Vibration Suppression Using Electromagnetic Resonant Shunt Damper. J Vib Acoust 2008;130(4):041003. doi:10.1115/1.2889916. 
[23] Zhu S, Shen WA, Qian X. Dynamic analogy between an electromagnetic shunt damper and a tuned mass damper. Smart Mater Struct 2013;22(11):115018. doi:10.1088/0964-1726/22/11/115018.

[24] McDaid AJ, Mace BR. A self-tuning electromagnetic vibration absorber with adaptive shunt electronics. Smart Mater Struct 2013;22(10):105013. doi:10.1088/0964-1726/22/10/105013.

[25] Paulitsch C, Gardonio P, Elliott SJ. Active vibration damping using a reactive, self-sensing, electrodynamic actuator. Smart Mater Struct 2006;15(2):499-508. doi:10.1088/0964-1726/15/2/033.

[26] Niu H, Zhang X, Xie S, Wang P. A new electromagnetic shunt damping treatment and vibration control of beam structures. Smart Mater Struct 2009;18(4):045009. doi:10 .1088/0964-1726/18/4/045009.

[27] Wang P, Zhang X. Damper based on electromagnetic shunt damping method. Int J Appl Electromagn Mech 2010;33(3/4):1425-30. doi:10.3233/JAE-2010-1270.

[28] Zhang X, Niu H, Yan B. A novel multimode negative inductance negative resistance shunted electromagnetic damping and its application on a cantilever plate. J Sound Vib 2012;331(10):2257-71. doi:10.1016/j.jsv.2011.12.028.

[29] Yan B, Zhang X, Niu H. Vibration isolation of a beam via negative resistance electromagnetic shunt dampers. J Intell Mater Syst Struct 2012;23(6):665-73. doi:10.1177/1045389X12437889.

[30] Yan B, Zhang X, Niu H. Design and test of a novel isolator with negative resistance electromagnetic shunt damping. Smart Mater Struct 2012;21(3):035003. doi:10 .1088/0964-1726/21/3/035003.

[31] Behrens S, Fleming AJ, Moheimani SOR. Passive Vibration Control via Electromagnetic Shunt Damping. IEEE/ASME Trans Mechatronics 2005;10(1):118-22. doi:10.1109/TMECH . 2004 . 835341. 
[32] Fleming AJ, Moheimani SOR, Behrens S. Synthesis and Implementation of Sensor-Less Active Shunt Controllers for Electromagnetically Actuated Systems. IEEE Trans Control Syst Technol 2005;13(2):246-61. doi:10.1109/TCST. 2004.839565.

[33] Fleming AJ, Moheimani SOR. Inertial vibration control using a shunted electromagnetic transducer. IEEE/ASME Trans Mechatronics 2006;11(1):84-92. doi:10.1109/TMECH. 2005.863364.

[34] Niederberger D, Behrens S, Fleming AJ, Moheimani SOR, Morari M. Adaptive electromagnetic shunt damping. IEEE/ASME Trans Mechatronics 2006;11(1):103-8. doi:10.1109/TMECH . 2005. 859844.

[35] Cheng TH, Oh IK. Vibration Suppression of Flexible Beam Using Electromagnetic Shunt Damper. IEEE Trans Magn 2009;45(6):2758-61. doi:10.1109/TMAG. 2009.2020549.

[36] Cheng TH, Oh IK. A current-flowing electromagnetic shunt damper for multi-mode vibration control of cantilever beams. Smart Mater Struct 2009;18(9):095036. doi:10 .1088/0964-1726/18/9/095036.

[37] Jung JH, Cheng TH, Oh IK. Electromagnetic Synchronized Switch Damping for Vibration Control of Flexible Beams. IEEE/ASME Trans Mechatronics 2012;17(6):1031-8. doi:10.1109/TMECH.2011.2157934.

[38] Ikegame T, Takagi K, Inoue T, Jikuya I. Sensor-less parameter estimation of electromagnetic transducer and experimental verification. In: Proc SPIE 9431. San Diego, USA; 2015, p. 943138. doi:10.1117/12.2085115.

[39] Ikegame T, Takagi K, Inoue T, Jikuya I. Sensor-less parameter estimation of electromagnetic actuator for shunt damping (in Japanese). Trans JSME 2015;81(825):15-00006. doi:10.1299/trans jsme.15-00006.

[40] Ishizaki T, Kashima K, Imura Ji, Katoh A, Morita H, Aihara K. Distributed parameter modeling and finite-frequency loop-shaping of elec- 
tromagnetic molding machine. Control Eng Pract 2013;21(12):1735-43. doi:10.1016/j . conengprac.2013.08.003.

${ }_{470}$ [41] Smythe WR. Static and Dynamic Electricity. Third ed.; New York: McGraw-Hill; 1968. ISBN 0891169172.

[42] Hayt WH, Buck JA. Engineering Electromagnetics. Eighth ed.; New York: McGraw-Hill; 2011. ISBN 0073380660.

[43] Cheng DK. Field and Wave electromagnetics. London: Wesley; 1983. ISBN 0201128195 .

[44] Rohrer RA. Circuit Theory: An Introduction to the State Variable Approach. New York: McGraw-Hill; 1970. ISBN 0070535574.

[45] The MathWorks Inc. . Matlab Optimization Toolbox User's Guide, Version 7.4. Natick, MA; 2016.

${ }_{480}$ [46] Takagi K, Inoue T, Miyachi T. Electro-Magnetic Shunt Damping with a Digital Virtual Impedance Circuit (in Japanese). Trans JSME 2012;78(786):474-88. doi:10.1299/kikaic.78.474.

[47] The MathWorks Inc. . Matlab Simulink User's Guide, Version 8.7. Natick, MA; 2016.

[48] Abramowitz M, Stegun IA. Handbook of Mathematical Functions With Formulas, Graphs and Mathematical Tables. New York: Dover Publications; 1965. ISBN 0486612724. 